\title{
Selective androgen receptor modulators activate the canonical prostate cancer androgen receptor program and repress cancer growth
}

\author{
Michael D. Nyquist ${ }^{1}$, Lisa S. Ang, ${ }^{1}$ Alexandra Corella, ${ }^{1}$ Ilsa M. Coleman, ${ }^{1}$ Michael P. Meers, ${ }^{2}$ Anthony J. Christiani, ${ }^{3}$ Cordell Pierce, ${ }^{1}$ \\ Derek H. Janssens, ${ }^{2}$ Hannah E. Meade, ${ }^{1}$ Arnab Bose, ${ }^{1}$ Lauren Brady, ${ }^{1}$ Timothy Howard, ${ }^{1}$ Navonil De Sarkar, ${ }^{1}$ Sander B. Frank, ${ }^{1}$ \\ Ruth F. Dumpit, ${ }^{1}$ James T. Dalton, ${ }^{4}$ Eva Corey, ${ }^{5}$ Stephen R. Plymate, ${ }^{3,6}$ Michael C. Haffner, ${ }^{1,7}$ Elahe A. Mostaghel, ${ }^{3,6,7}$ \\ and Peter S. Nelson ${ }^{1,5,6,7}$ \\ Division of Human Biology and 'Division of Basic Sciences, Fred Hutchinson Cancer Research Center, Seattle, Washington, USA. 'Ceriatric Research, Education, and Clinical Center, VA Puget Sound Health \\ Care System, Seattle, Washington, USA. ${ }^{4}$ Office for Academic Affairs, University of Alabama, Tuscaloosa, Alabama, USA. ${ }^{5}$ Department of Urology and ${ }^{6}$ Department of Medicine, University of Washington, \\ Seattle, Washington, USA. Division of Clinical Research, Fred Hutchinson Cancer Research Center, Seattle, Washington, USA.
}

Prostate cancer (PC) is driven by androgen receptor (AR) activity, a master regulator of prostate development and homeostasis. Frontline therapies for metastatic PC deprive the AR of the activating ligands testosterone $(T)$ and dihydrotestosterone (DHT) by limiting their biosynthesis or blocking AR binding. Notably, AR signaling is dichotomous, inducing growth at lower activity levels, while suppressing growth at higher levels. Recent clinical studies have exploited this effect by administration of supraphysiological concentrations of $\mathrm{T}$, resulting in clinical responses and improvements in quality of life. However, the use of $\mathrm{T}$ as a therapeutic agent in oncology is limited by poor drug-like properties as well as rapid and variable metabolism. Here, we investigated the antitumor effects of selective AR modulators (SARMs), which are small-molecule nonsteroidal AR agonists developed to treat muscle wasting and cachexia. Several orally administered SARMs activated the AR program in PC models. AR cistromes regulated by steroidal androgens and SARMs were superimposable. Coregulatory proteins including HOXB13 and GRHL2 comprised AR complexes assembled by both androgens and SARMs. At bioavailable concentrations, SARMs repressed MYC oncoprotein expression and inhibited the growth of castration-sensitive and castration-resistant $P C$ in vitro and in vivo. These results support further clinical investigation of SARMs for treating advanced PC.

\section{Introduction}

Prostate secretory epithelial cells and neoplastic derivatives are dependent on the androgen receptor (AR) to regulate differentiation, metabolism, growth, and survival (1-3). Currently, inhibition of AR signaling using androgen deprivation therapy (ADT), which involves various means of depriving tumors of the AR ligands testosterone (T) and dihydrotestosterone (DHT), is the predominant therapeutic approach for treating metastatic prostate cancer (mPC) (4). Although ADT is highly effective initially, $\mathrm{mPC}$ progresses to metastatic castration-resistant prostate cancer (mCPRC) within 1 to 3 years (5).

The mechanisms of resistance to ADT are diverse. The majority of mCRPCs reactivate AR signaling through $A R$ amplification, the production of AR splice variants, $A R$ mutation, or

Conflict of interest: JTD reports receiving royalty payments related to patents for SARM development and royalty distributions from the University of Tennessee Research Foundation related to SARM patents (US 2019/0055192 A1). PSN has served as an advisor to Astellas Pharma, Janssen Pharmaceuticals, and Bristol Myers Squibb. Copyright: () 2021, American Society for Clinical Investigation.

Submitted: December 9, 2020; Accepted: March 31, 2021; Published: May 17, 2021

Reference information: J Clin Invest. 2021;131(10):e146777.

https://doi.org/10.1172/JCl146777. changes in AR cofactors (5). Although retargeting AR signaling in $\mathrm{mCRPC}$ with potent AR antagonists such as enzalutamide (ENZ) or CYP17A inhibitors such as abiraterone (ABI) extends survival, responses to these treatments average only a few months $(6,7)$. Long-term inhibition of AR activity can result in significant quality-of-life complications related to decreased muscle mass, reduced bone density, erectile dysfunction, anemia, increased body fat, cardiovascular events, and hot flashes $(8,9)$. Further, emerging evidence indicates that potent suppression of AR signaling may promote transdifferentiation into highly aggressive variants including those with small-cell and neuroendocrine features.

Developing new therapeutic approaches that take advantage of the therapeutic window provided by the AR, while maintaining quality of life, could have a substantial clinical impact. Supraphysiological androgen therapy (SAT) is one approach that is potentially capable of achieving a dual benefit. Although controversial and somewhat counterintuitive as a treatment strategy, SAT builds upon longstanding in vitro experiments demonstrating that high androgen concentrations induce cell-cycle arrest in AR-active PC cells $(10,11)$. SAT has been evaluated clinically by treating patients with monthly cycles of high-dose $\mathrm{T}$ therapy alternated with low androgen concentrations, a strategy termed bipolar androgen 
A

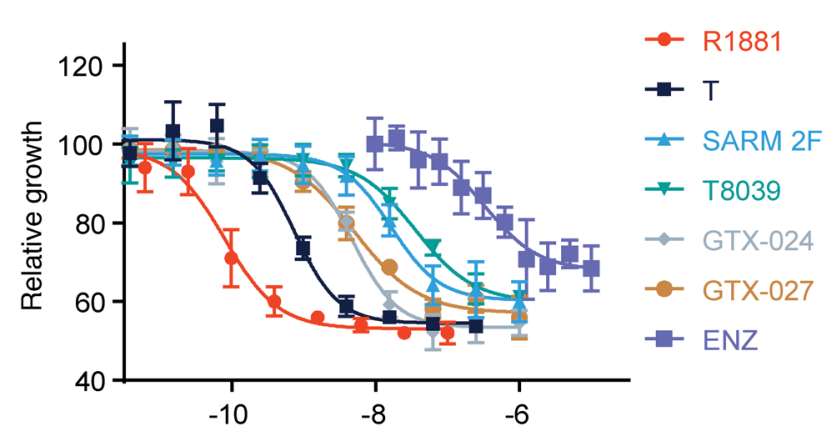

Concentration $\log _{10}(M)$

C

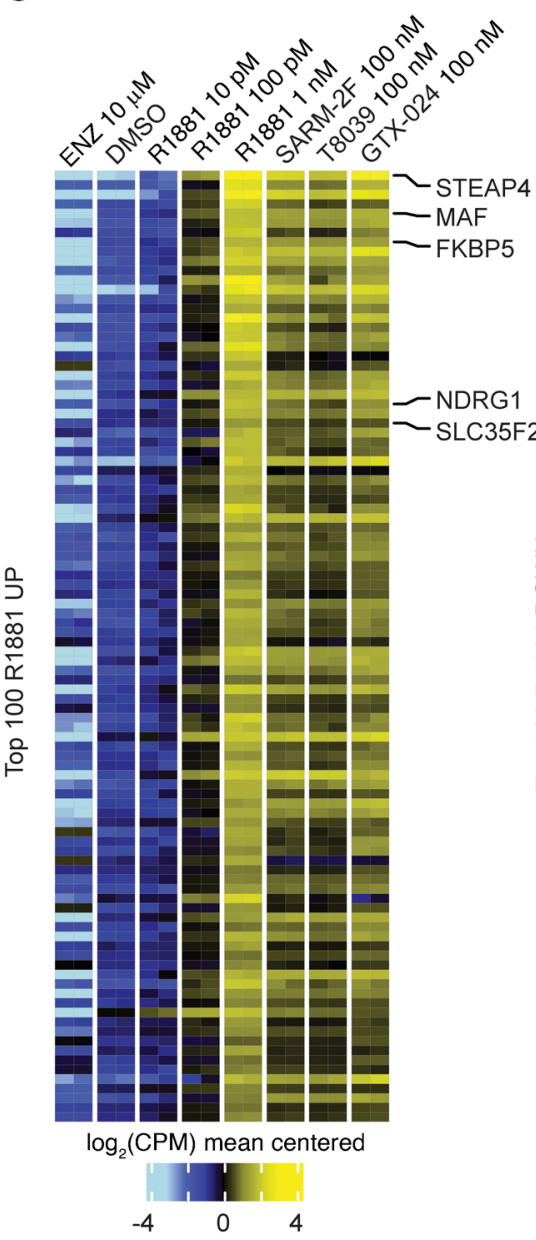

D

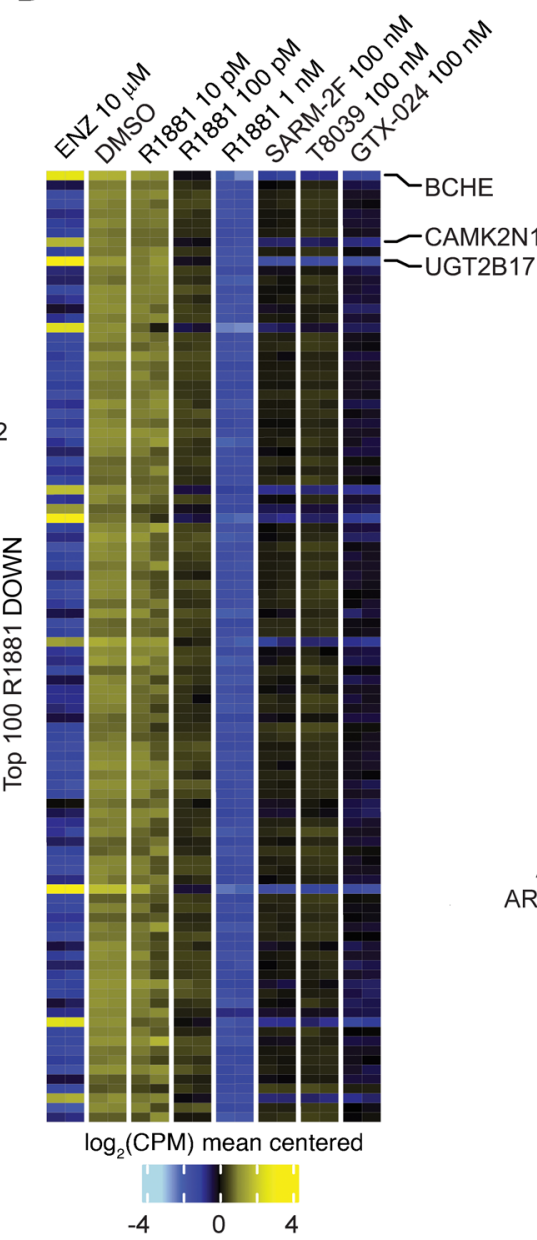

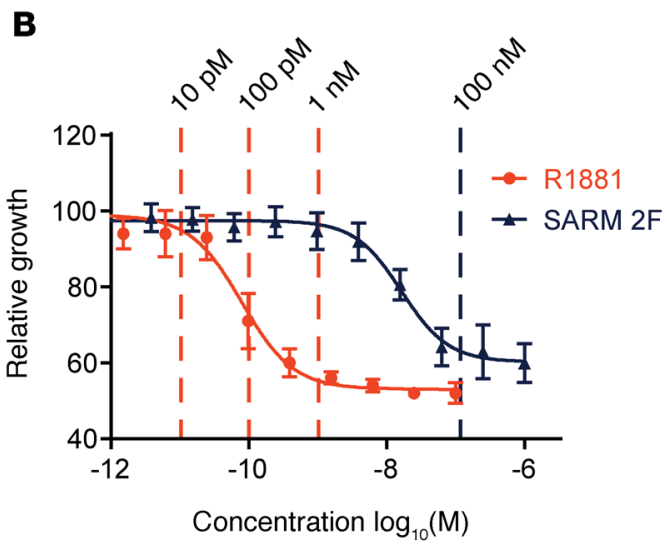

E

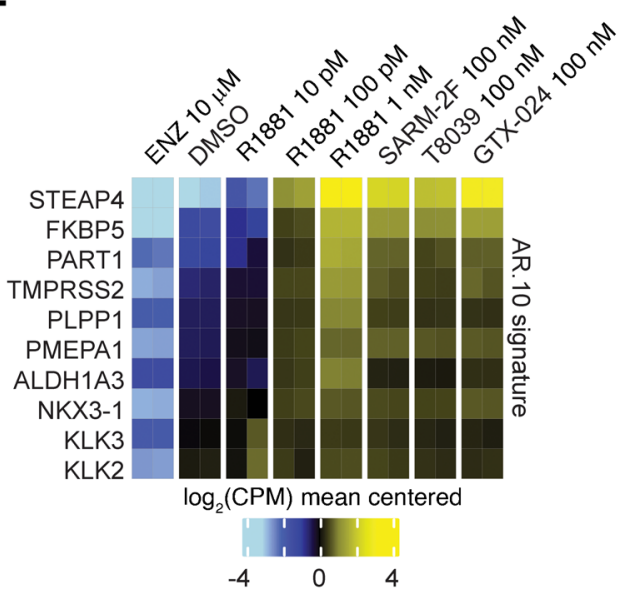

$\mathbf{F}$

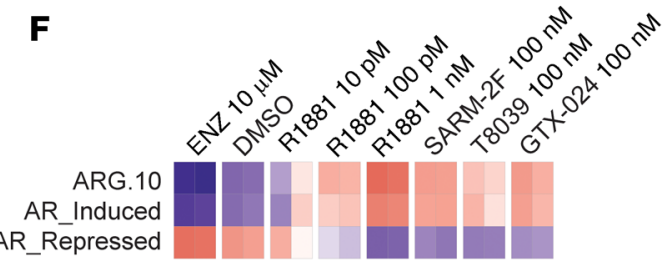

Figure 1. Selective AR modulators activate the canonical AR program in PC cells and repress tumor cell proliferation. (A) Dose-response curves were determined in the LNCaP cell line for the steroidal androgens R1881 and T, the antiandrogen ENZ, and the nonsteroidal AR agonists T8039, GTX-024, GTX-027, and SARM-2F $(n=4)$. Data represent the mean \pm SD. (B) Doses of R1881 and SARMs chosen for RNA-Seq analysis. Gene expression heatmaps are shown for the top 100 genes (C) induced or (D) repressed by treatment with $1 \mathrm{nM}$ R1881 $(n=2)$. (E) Gene expression heatmap of genes that comprise the ARG.10 signature. (F) GSVA signature score heatmap for the AR-regulated gene sets ARG.10, AR_Induced, and AR_Repressed.

therapy (BAT). BAT has shown promise both in efficacy and safety, with approximately $30 \%$ response rates in heavily pretreated patients with mCPRC who progressed on ENZ (12). Furthermore, in some patients, BAT resensitizes mCRPCs to retreatment with antiandrogen therapy and improves quality-of-life metrics (1214). Given the ability of mCRPC to adapt to static androgen levels over time, the dynamism of this therapy may be crucial in preventing or delaying the genetic and epigenetic changes that engender a therapy-resistant state of disease.

The potential of BAT notwithstanding, there are significant drawbacks to using $\mathrm{T}$ as a therapeutic reagent. $\mathrm{T}$ has poor oral bioavailability and drug-like properties and is metabolized into 
various compounds such as DHT, estradiol, and other derivatives with biological activity that could induce unwanted side effects. Because of its rapid metabolism, it is challenging to control $\mathrm{T}$ levels and establish optimized dosing regimens. The administration of $\mathrm{T}$ relies on transdermal gels or i.m. injections, and high doses of steroidal androgens may result in organ damage, including hepatotoxicity, erythrocytosis, and cardiovascular complications, as well as unfavorable lipogenic activities by lowering HDL and raising triglycerides (15-18). Given these issues, alternatives to $\mathrm{T}$ that induce the tumor-suppressive functions of the AR while bypassing the deleterious androgenic effects of steroids may be ideal therapeutics.

Selective androgen receptor modulators (SARMs) were developed as a substitute for steroidal androgens to treat a range of conditions including partial androgen insensitivity syndrome (PAIS), cachexia, age-related sarcopenia, osteoporosis, and certain muscular dystrophies (19-21). Molecules that engage the ligand-binding domain of the AR fall along the spectrum of antagonist to full agonist. To improve safety and tolerability, SARMs were designed to be partial AR agonists, allowing anabolic functions that support systemic health while mitigating the androgenic functions of $\mathrm{T}$ that induce unwanted side effects including the potential to promote prostate pathology $(20,21)$. Clinical and preclinical studies demonstrated that SARMs can support muscle mass without prostate hypertrophy or alterations in secondary sexual characteristics (19, 22-25). Nonsteroidal SARMs also have favorable drug properties and can be delivered via oral $(23,26)$ or transdermal routes (27). Furthermore, the pharmacological characteristics of nonsteroidal agonists allow for improved safety and greater control over the timing and dosage $(19,25,26,28)$. Unlike T, SARMs are not substrates of metabolizing enzymes such as $5 \alpha$-reductase, $17 \beta-H S D$, and CYP19.

In this study, we sought to test the hypothesis that SARMs can repress the growth of PC by activating the canonical AR program and consequently substitute for steroidal androgens as therapeutics with favorable pharmacokinetic and pharmacodynamic properties.

\section{Results}

SARMs and androgens repress the growth of PC cells and induce concordant transcriptional changes. Several studies have demonstrated that high concentrations of T can suppress the growth of PC cells in vitro and in vivo $(14,29-32)$. We sought to determine whether nonsteroidal and orally bioavailable SARMs can exert similar effects. We selected several SARMs that had progressed through various stages of preclinical or clinical evaluation and demonstrated physiological effects and a lack of toxicity. GTX-024, also known as enobosarm, has been extensively studied in humans, with clinical trials evaluating the effects on cancer cachexia and muscle wasting (33). GTX-024 and a related SARM, GTX-027, suppressed the growth of triple-negative breast cancer (TNBC) xenografts without adverse effects on animal health (34). GTX-024 has been evaluated in phase II clinical trials of AR-positive breast cancer (NCT02971761; NCT02368691), with no reported adverse safety signals (35). SARM-2F was shown to support muscle, prostate, and seminal vesicle mass in rats following castration and improved outcomes in a model of cancer cachexia (23). Furthermore, SARM-2F increased lean body mass in primates and was well tol- erated (19). The SARM compound-26 (Cpd26) has been shown to potently activate the AR and support anabolic growth in vivo and can be administered via the transdermal route (27).

We treated the androgen-responsive LNCaP PC cell line with concentration ranges of several SARMs: GTX-024, GTX-027, SARM-2F, and T8039. We compared the SARMs with 2 steroidal agonists of AR, T and R1881, as well as with the AR antagonist ENZ. All 4 SARMs suppressed LNCaP cell growth by approximately $40 \%$ relative to the control $(P<0.001)$ and achieved their maximum effect at approximately $100 \mathrm{nM}$. In comparison, R1881 and T produced maximal growth suppression of approximately $50 \%$ at approximately $1 \mathrm{nM}\left(P=6.6 \times 10^{-7}\right)$ and approximately $10 \mathrm{nM}(P=$ $\left.6.2 \times 10^{-8}\right)$, respectively (Figure $\left.1 \mathrm{~A}\right)$. ENZ concentrations of $1 \mu \mathrm{M}$ produced maximal growth repression of $\mathrm{LNCaP}$ cells, reducing cell viability by $32 \%$ compared with vehicle control $\left(P=5.0 \times 10^{-5}\right)$, in agreement with previous studies of ENZ in this model (Figure $1 \mathrm{~A}$ and refs. 36, 37). Two other SARMs, lgd-4033 and RAD-140, failed to suppress the growth of LNCaP cells (Supplemental Figure 1, A and B; supplemental material available online with this article; https://doi.org/10.1172/JCI146777DS1), indicating that molecular differences in SARM structures influence cellular responses (Supplemental Figure 2, A-F).

SARMs were developed to be partial agonists in order to minimize androgenic AR activation in the prostate. However, SARMs can act as AR antagonists in certain contexts and interfere with AR activation. To determine whether the growth-suppressive effects of SARMs are due to activation or suppression of AR signaling, we measured global transcript levels by RNA-Seq in LNCaP cells treated for 48 hours with 100 nM SARM-2F, T8039, or GTX-024. These SARM concentrations produced maximum growth suppression of LNCaP cells in vitro (Figure 1B), and levels exceeding $100 \mathrm{nM}$ have been achieved in rodents, primates, and humans without toxicities $(19,38)$. As a comparison, we also performed RNA-Seq on LNCaP cells treated for 48 hours with a dose range of $10 \mathrm{pM}, 100 \mathrm{pM}$, and $1 \mathrm{nM} \mathrm{R} 1881$ to measure the transcriptional changes corresponding to no, intermediate, and maximal growth suppression (Figure 1B). The genes upregulated to the greatest degree by $1 \mathrm{nM}$ R1881 were also strongly upregulated by each SARM, including the canonical AR target genes KLK3, FKBP5, STEAP4, MAF, NDRG1, and SLC25F2 (Figure 1C). As expected, these transcripts were repressed by ENZ treatment (Figure 1C). The genes most repressed by $1 \mathrm{nM} \mathrm{R} 1881$, including UGT2B17, BCHE, and CAMK2N1, were reduced to a similar degree by the SARMs (Figure 1D).

We used gene set variation analysis (GSVA) to confirm that SARM exposure altered gene expression pathways associated with prior studies of AR activity and/or androgen responses. Scores for gene signatures associated with AR activity - ARG.10, ARInduced, and AR-Repressed - were concordantly altered (Figure 1, E and F), suggesting that the growth-suppressive effects of SARMs reflect activation rather than repression of AR signaling. Using an orthogonal assay, we confirmed the changes in expression levels of the canonical AR target genes FKBP5, KLK3, and $A M A C R$ by quantitative reverse transcription PCR (qRT-PCRs) in LNCaP and VCAP cells (Supplemental Figure 3, A-F) and observed transcriptional changes at SARM concentrations that repressed cell growth (Supplemental Figure 3, G-I). 
A
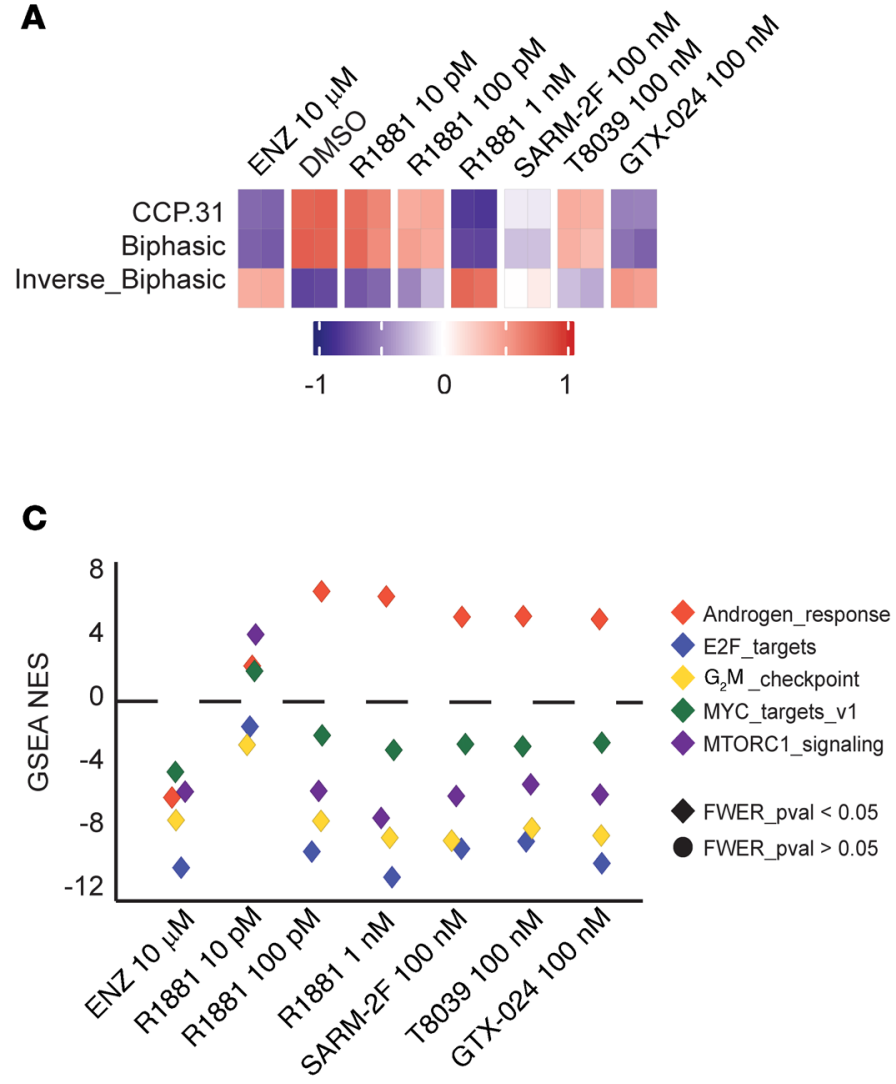

D

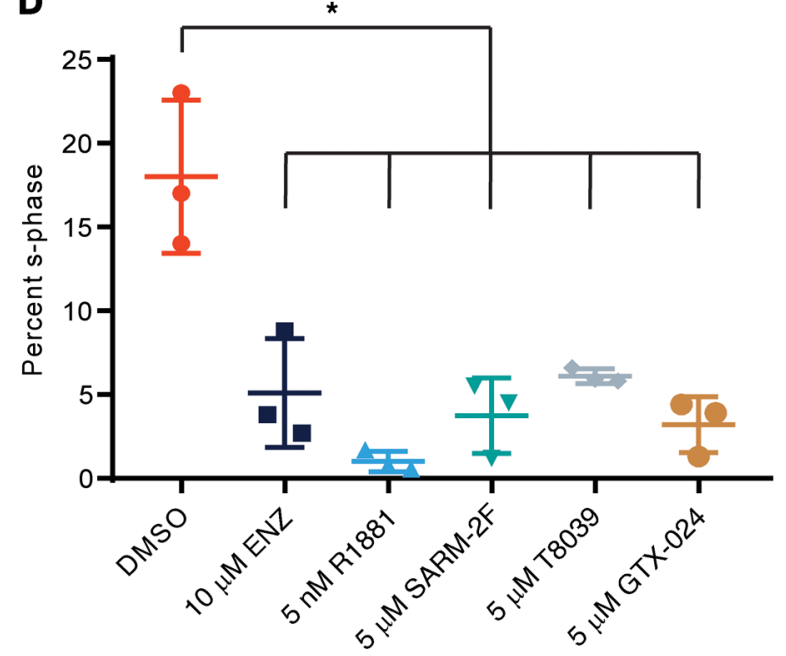

B

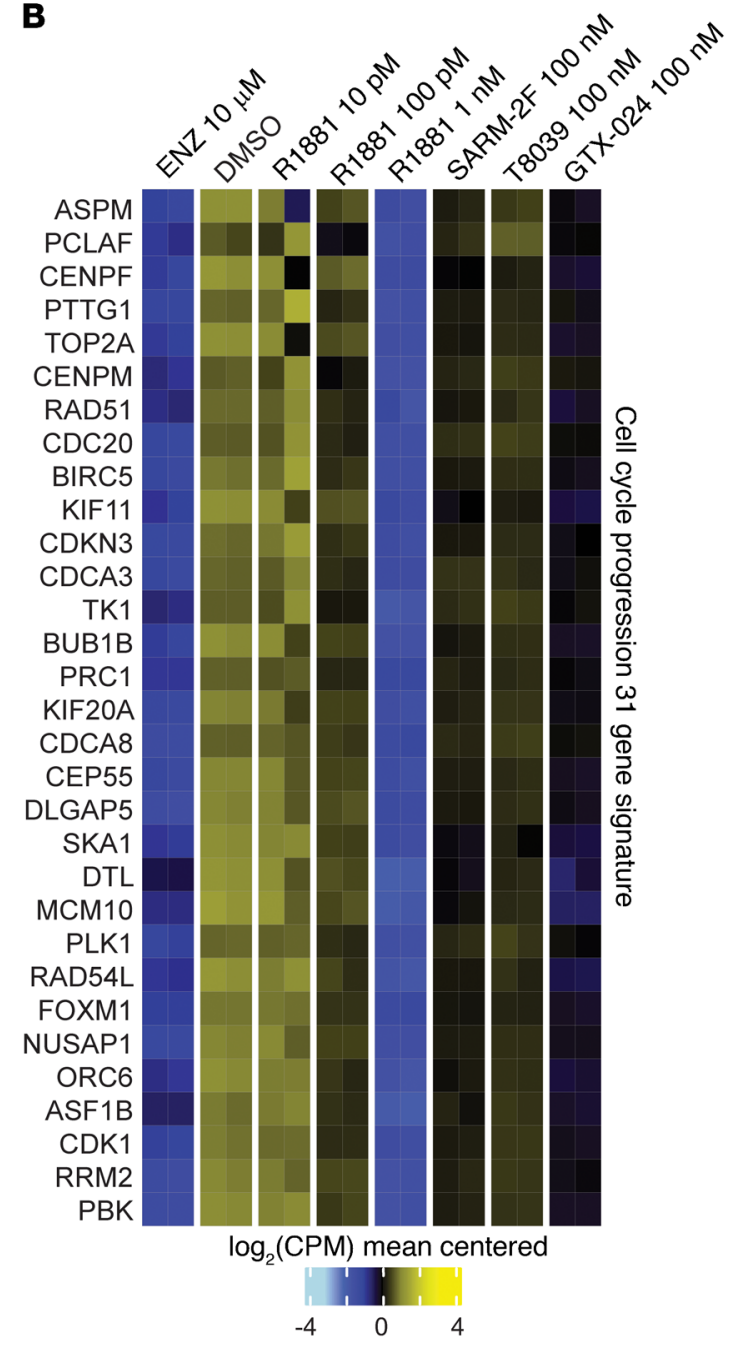

$\mathbf{E}$

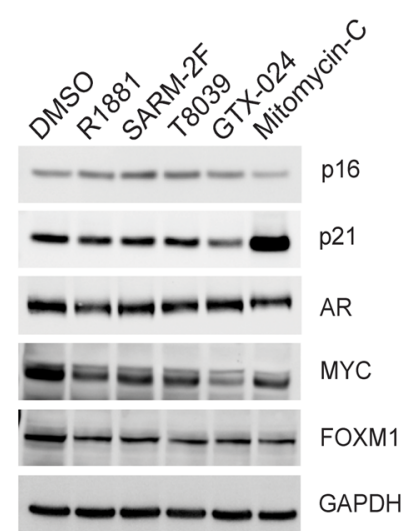

Figure 2. SARMs suppress MYC levels and proliferation-associated gene expression. (A) GSVA signature score heatmap of cell-cycle-related gene sets from RNA-Seq data. (B) Heatmap of RNA-Seq mean-centered $\log _{2}$ (CPM) values for cell-cycle progression signature genes. (C) CSEA normalized enrichment scores (NESs) plotted for Hallmark gene sets. (D) Percentage of LNCaP cells in S phase when treated for 48 hours with $10 \mu M$ ENZ, 5 nM R1881, or $5 \mu M$ SARMs ( $n$ $=3$ ). ${ }^{*} P<0.05$, by 1 -way ANOVA with Sidak's multiple-comparison test. Data represent the mean \pm SD. (E) Immunoblots for p16, p21, AR, FOXM1, and MYC performed on LNCaP cells treated with $5 \mathrm{nM}$ R1881, $5 \mu \mathrm{M}$ SARMs, or $0.4 \mu \mathrm{g} / \mathrm{mL}$ mitomycin-C for 6 days.

SARM treatment induces cell-cycle growth arrest and represses MYC expression. Previous studies have demonstrated that proliferation-associated gene expression changes reflect the efficacy of high-dose androgen exposure (29). To determine whether similar changes occur with SARM treatment, we performed GSVA on the RNA-Seq data sets to evaluate the activity of gene sets associated with proliferation and cell-cycle activity. These included 31 genes comprising a cell-cycle progression score (CCP.31) (39) and genes 
that exhibit biphasic responses to androgens, whereby expression was low in quiescent cells in the absence of androgens, increased with the induction of proliferation at low androgen concentrations, and then decreased with high concentrations of androgen (biphasic) concurrent with growth arrest (29). Changes induced by SARM treatment were concordant with responses to concentrations of $10 \mathrm{pM}$ and $100 \mathrm{pM}$ R1881 for T8309, and $1 \mathrm{nM}$ R1881 for SARM-2F and GTX-024 (Figure 2, A and B). Next, using the HALLMARK gene set collection, we performed gene set enrichment analysis (GSEA) on the RNA-Seq measurements from cells treated with ENZ, R1881, and SARMs. To identify the gene sets of highest relevance to the growth-suppressed phenotype, we compared the highest dose of R1881 that supports full proliferation, $10 \mathrm{pM}$, with a concentration that suppressed proliferation, 100 pM R1881 (Supplemental Figure 4A). Consistent with previous reports, the most repressed gene sets were HALLMARK_E2F_TARGETS and HALLMARK_MYC_TARGETS_V1 (29). SARMs potently suppressed E2F and MYC pathway gene expression as well as genes associated with the $\mathrm{G}_{2} / \mathrm{M}$ checkpoint (Figure $2 \mathrm{C}$ ).

To further evaluate the effects of SARMs on cell proliferation, we performed cell-cycle analysis using a 1-hour pulse of EdU as an S-phase marker in LNCaP cells treated for 48 hours with $5 \mathrm{nM}$ R1881, $10 \mu \mathrm{M}$ ENZ, or $5 \mu \mathrm{M}$ SARM-2F, GTX-024, or T8039. All treatments reduced the number of cells in S-phase from approximately $17 \%$ to $2 \%-5 \%(P<0.05$; Figure $2 D)$. After 6 days of SARM treatment, we observed no increase in p16 or p21 levels, indicating that the cells were not in a senescent state (Figure 2E). AR protein levels in LNCaP cells were not dramatically changed with SARM treatment (Figure 2E). Previous studies have reported that supraphysiological androgen levels repress MYC, and we determined that SARM exposure also reduced MYC levels in LNCaP cells (ref. 40 and Figure 2E). Two transcription factors, FOXM1 and $M Y B L 2$, which are known to be repressed by the dimerization partner, RB-like, E2F and multi-vulval class B (DREAM) complex and involved in regulating quiescence and the gene ontology (GO) cell-cycle phase, were reduced by SARM treatment in LNCaP and VCaP cells (ref. 41, Figure 2E, and Supplemental Figure 5, A-D).

In contrast to orally available SARMs, transdermal formulations have the advantage of bypassing the first-pass oral-hepatic metabolism, which could yield further improvements to safety and efficacy $(27,42)$. We evaluated the effects of one such SARM, Cpd26 (27), on CRPC models. Cpd26 potently suppressed the growth of LNCaP, VCaP, and 22PC-EP cells (Figure 3A). Cpd26 also suppressed MYC and FOXM1 levels, but had no effect on p21 (Figure 3B). Expression of the androgen-induced genes $K L K 3$, FKBP5, and AMACR increased with Cpd26 treatment, with saturating effects occurring near $1 \mathrm{nM}$ (Figure 3, C and D). The androgen-repressed gene UGT2B17 (Figure 3E) and cell-cyclerelated genes CDK1, FOXM1, and MYBL2 were also repressed in a dose-dependent manner (Figure 3, F-H). Similar transcriptional changes were induced by Cpd26 in VCaP cells expressing a WT AR (Supplemental Figure 6, A-F).

PC AR cistromes regulated by SARMs and androgens are concordant. To compare how steroidal androgens and nonsteroidal SARMs specify the localization of the AR to chromatin, we evaluated AR DNA-binding sites genome wide with the cleavage under targets and release using the nuclease (CUT\&RUN) method following exposure of LNCaP cells to R1881, T8039, SARM-2F, and GTX024. SARM-induced AR peaks were highly similar to those induced by R1881 (Figure 4A). We found that SARM-liganded AR-binding sites were associated with canonical AR targets including $K L K 3$ (Figure 4B) and FKBP5 (Figure 4C). Globally, SARM and R1881 peak sets annotated to highly similar genomic features. Moreover, motif analysis established that SARMs, like R1881, induced binding to androgen response elements (AREs) as well as to established cofactor motifs for FOXA1 and HOXB13 (Figure 4D).

High-dose androgen and high-dose SARM induced AR binding to overlapping sites. Overall, the R1881 and SARM cistromes were highly similar: 9320 of 11,287 (83\%) sites gained with SARM treatment overlapped with those of high-concentration R1881 (Figure 5A). While the R1881 and SARM AR cistromes largely overlapped, there were 952 differentially bound sites between SARMs and R1881, and, of these, 96\% were gained in the R1881-treated cells (Figure 5B). These differences were also reflected by principal component analysis (PCA), with R1881 replicates grouping away from the SARM samples (Supplemental Figure 7B). We hypothesized that the additional R1881 AR sites represent genomic loci with lower AR affinity and would be lost with lower concentrations of R1881. To explore this, we performed CUT\&RUN using $5 \mathrm{nM}$, $1 \mathrm{nM}, 100 \mathrm{pM}$, and $10 \mathrm{pM}$ R1881 to determine sites lost or gained with reduced AR agonism. Compared with the low-dose groups (100 pM, 10 pM, and DMSO), we identified 11,859 sites that were differentially bound with high-dose R1881 (5 nM and $1 \mathrm{nM}$, Supplemental Figure 7C) and the R1881 dose groups associated by their level of agonism by PCA (Supplemental Figure 7D). The AR-binding profiles induced by high-dose SARMs clustered with the high-dose R1881 groups (Figure 5C), and 910 of the 952 sites that were differentially bound between SARMs and R1881 were also differentially bound between low and high doses of R1881 (Figure 5D). Taken together, these data suggest that the small subset of AR sites that were differentially bound between R1881 and SARM treatment were due to a slightly lower level of agonism.

SARMs induce the recruitment of known AR cofactors. SARMs have been reported to selectively recruit AR cofactors to explain the different effects SARMs have on the prostate versus bone and muscle tissue $(21,43)$. By identifying the cofactors that are shared or not shared by steroidal androgens and SARMs, we sought to determine which factors are essential for the growth-suppressive effects of SARMs. We used the Microarray Assay for Realtime Coregulator Nuclear receptor Integration (MARCoNI) platform to measure ligand-induced binding of the AR ligand-binding domain (LBD) with the interaction domain of a panel of nuclear receptor peptides immobilized on a solid support (44). The binding of the liganded AR LBD was measured with saturating doses $(10 \mu \mathrm{M})$ of SARM-2F, GFX-024, GTX-027, T8039, and Cpd26 and compared against the steroidal androgen DHT and DMSO as positive and negative controls, respectively. DHT induced AR-LBD binding to known AR cofactors including NCOA1, NCOA3, PIAS2, p300, and CBP over the level of DMSO (Supplemental Figure 8A). In contrast, SARMs were unable to consistently support these associations (Supplemental Figure 8B), even for cofactors very highly enriched with DHT (Supplemental Figure 8C).

The lack of SARM-mediated AR-LBD and cofactor interactions in the MARCoNI assay suggested either a limitation of the MARCoNI 
A

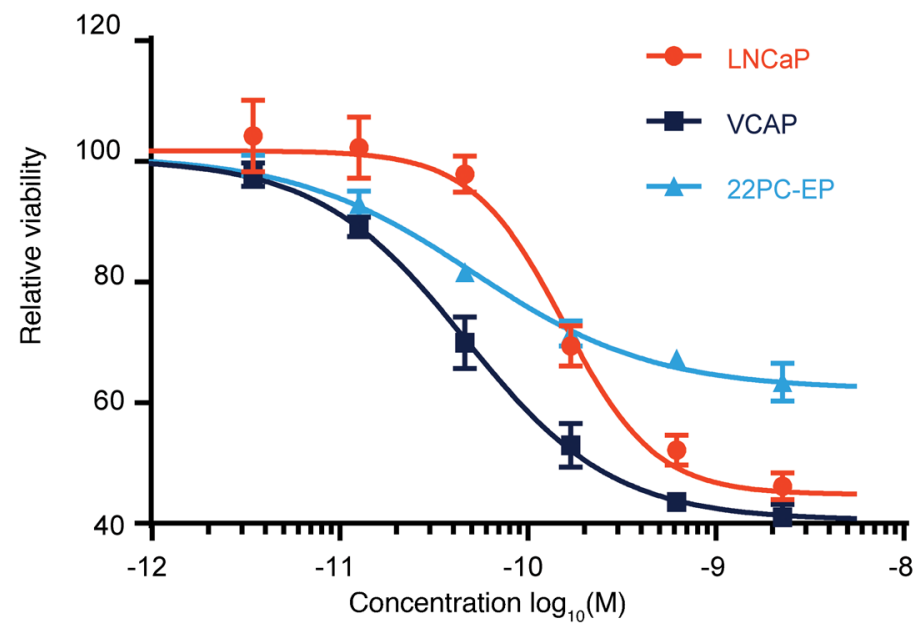

B

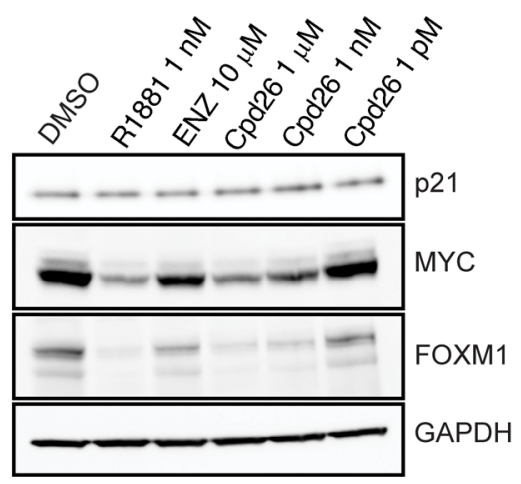

E

$\mathbf{F}$

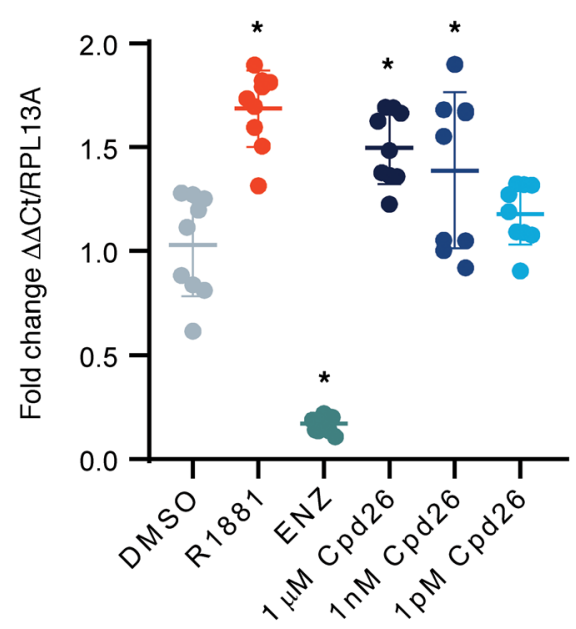

CDK1

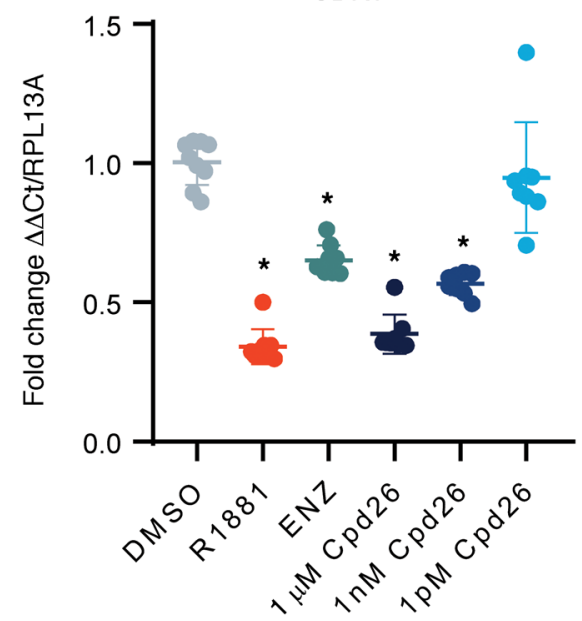

FKBP5

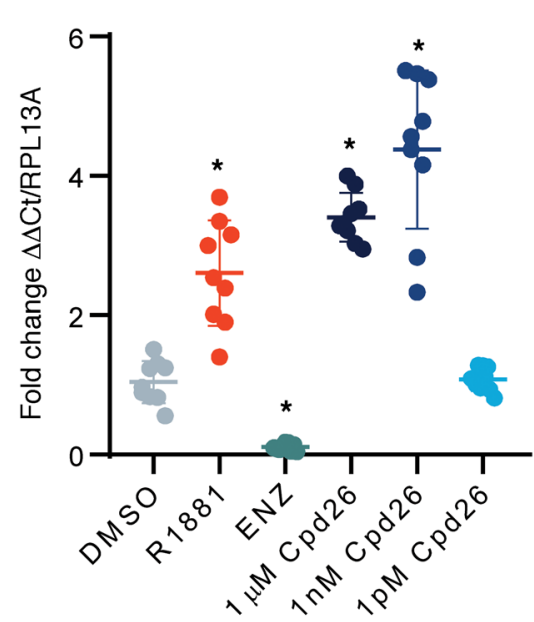

G

D

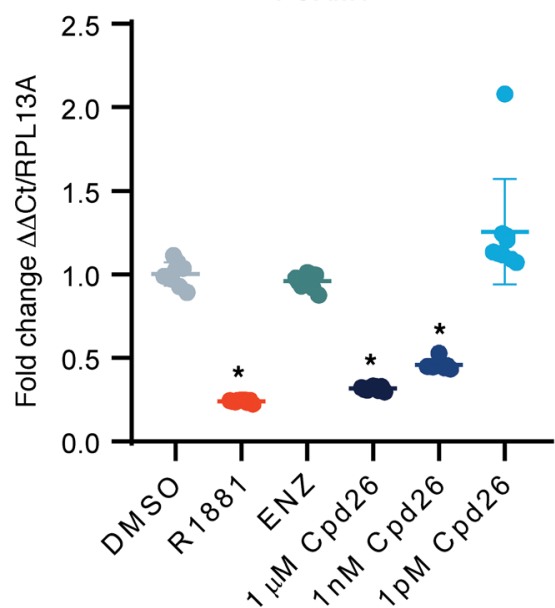

UGT2B17

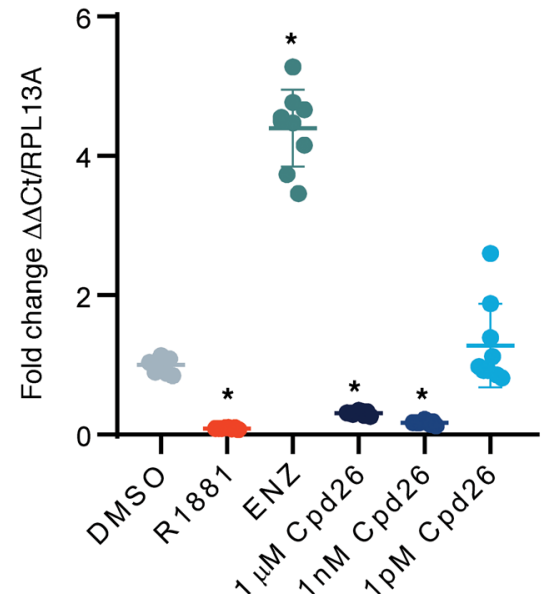

H

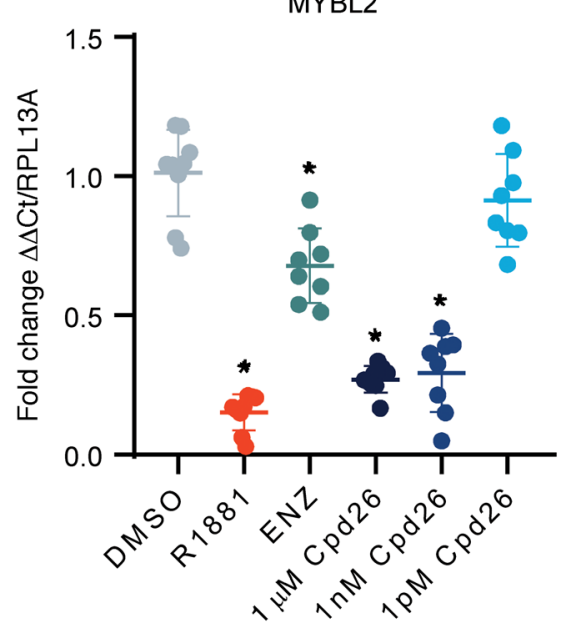

Figure 3. The SARM Cpd26 potently suppresses PC growth and induces luminal epithelial gene expression. (A) Dose-response curve of the transdermal SARM Cpd26 in LNCaP, VCaP, and 22PC-EP cells $(n=4)$. Data represent the mean \pm SD. (B) Immunoblots of the cell-cycle proteins p21, MYC, and FOXM1 in LNCaP cells treated with R1881, ENZ, or Cpd26. qRT-PCR values are plotted for LNCaP cells treated with ENZ, R1881, or doses of Cpd26 for 48 hours, measuring the expression of the AR-activated genes (C) KLK3 and (D) FKBP5; the AR-repressed gene (E) UGT2B17; and the cell-cycle genes (F) CDK1, (G) FOXM1, and $(\mathbf{H})$ MYBL2 $(n=8) .{ }^{*} P<0.05$, by 1-way ANOVA with Dunnett's multiple-comparison test. Data represent the mean \pm SD. 


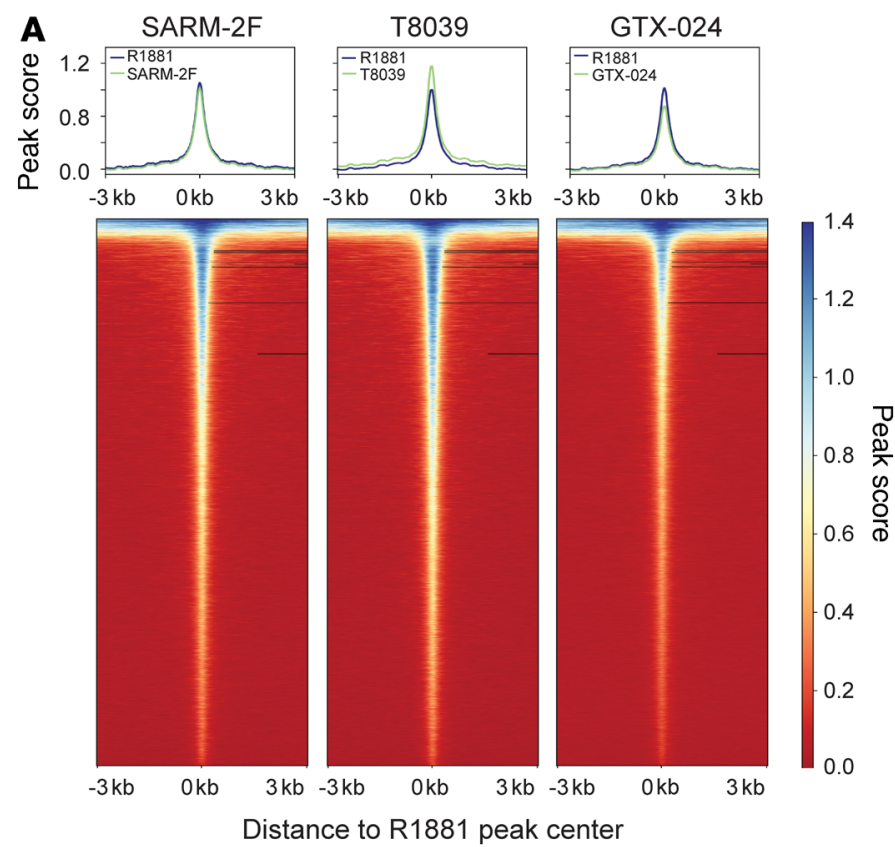

D

\begin{tabular}{|c|c|c|c|c|c|}
\hline \multicolumn{2}{|c|}{ Motif } & R1881 & SARM-2F & T8039 & GTX-024 \\
\hline ARE & & $P=1 \mathrm{e}-531$ & $P=1 \mathrm{e}-546$ & $=1$ & $=1 \mathrm{e}-686$ \\
\hline FOXA1 & & $P=1 \mathrm{e}-598$ & $P=1 \mathrm{e}-670$ & $P=1$ & 17 \\
\hline HOXB13 & ITTTAIT & $P=1 \mathrm{e}-149$ & $P=1 \mathrm{e}-210$ & $P=1 \mathrm{e}-334$ & $P=1 \mathrm{e}-2$ \\
\hline
\end{tabular}

B

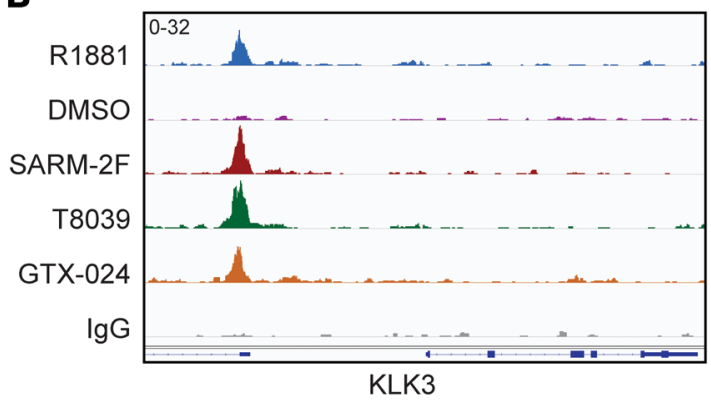

C

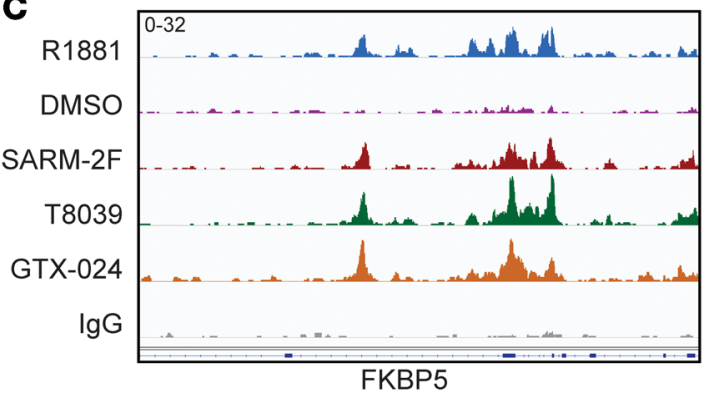

Figure 4. SARMs and steroidal androgens regulate concordant AR cistromes in PC. (A) Heatmap of SARM-induced AR binding to R1881induced AR binding sites determined by CUT\&RUN. Comparison of SARM and steroid-induced AR binding near (B) KLK3 and (C) FKBP5 (peaks are representative of 2 biological replicates). (D) Motif analysis of AR-bound sites across R1881 and SARM treatments (for all groups, $q<0.0001$ ). assay to evaluate nonsteroidal agonists, or that AR cofactor interactions important for SARM activity may occur via other regions of the AR such as the N-terminal AF1 domain or through other cell-intrinsic factors. Moreover, in vitro binding assays do not model interactions with most endogenous proteins, nor do they account for the expression levels of these proteins. Further, other in vitro assays such as those using LXXLL-AF2 or AR N-/C-terminal interaction reporters poorly predicted the effects of steroidal SARM molecules in PC cells $(45,46)$. For this reason, we performed rapid immunoprecipitation mass spectrometry of endogenous proteins (RIME) to identify proteins in AR complexes on chromatin (47). Briefly, we treated LNCaP cells with $5 \mu \mathrm{M}$ SARM-2F or 5 nM R1881 for 4 hours. Chromatin complexes were isolated, immunoprecipitated with an AR antibody, and analyzed by mass spectrometry. RIME quantitation identified 321 and 249 proteins that were significantly enriched over IgG controls in 3 replicates of SARM-2F- and R1881-treated samples, respectively (FDR $q<0.05$; Figure 6A). Of these, 246 proteins overlapped between SARM-2F and R1881 and were similarly enriched in the SARM-2F and R1881 groups (Figure 6B).

We evaluated the known functions of the 246 overlapping proteins identified in the RIME analysis of SARM-2F- and R1881-treated samples (Figure 6A). The most enriched functional category within the shared proteins by GO analysis was "nucleic acid metabolism protein" (Supplemental Figure 8D). We found that AR cofactors critical for luminal differentiation, including HOXB13, GRHL2, UTY, and NKX3-1, were bound to the AR at similar levels in the SARM-2F- and R1881-treated samples (Figure 6C). Both SARM-2F and R1881 induced the AR to associate with transcription factors and chromatin modifiers, both activating and repressing, including EP300 and KDM1A (Figure 6D), SWI/SNF complex members (Figure 6E), and DNA repair and replication components (Figure 6F). These data suggest that both the SARM2F- and R1881-liganded AR assembled highly similar complexes.

While previous studies suggested that SARMs may impart their tissue-selective effects by recruiting a subset of cofactors and differentially regulating gene expression (43), no protein was significantly enriched in R1881-treated samples over SARM-2F-treated samples or in SARM-2F- over R1881-treated samples (FDR $q<0.05$; Figure $6 \mathrm{~B}$ and Supplemental Table 1 ). The proteins with the most significant differences between SARM-2F and R1881 were MRPL19, MDH2, HBB, ACAT1, and CPSF7 (FDR $q>0.14$ ). These proteins are not known transcription factors and do not have validated interactions with the AR, suggesting that they represent nonspecific interactions with the AR antibody or the immunoprecipitated AR complex.

Growth-repressive effects on PC vary by $S A R M$ and require $A R$ activity. In addition to the growth-inhibitory effects of high SARM concentrations on LNCaP cells (Figure 1A), we determined that multiple SARMs also repressed the growth of AR-WT VCAP PC cells with potencies similar to those of $T$, reaching saturating effects at approximately $10 \mathrm{nM}$ (Figure 7A). SARM-2F and T8039 suppressed the growth of 22PC-EP PC cells, which harbor the $\mathrm{AR} \mathrm{H} 874 \mathrm{Y}$ mutation $\left(\mathrm{AR}^{\mathrm{H} 874 \mathrm{Y}}\right)$ to an extent similar to that seen with R1881, saturating at approximately $1 \mathrm{nM}$, a concentration slightly lower than the levels of $\mathrm{T}$ required to achieve similar growth repression (Figure 7B). While 22PC-EP cells were very sensitive to ENZ, the relative potency of ENZ was approximately 100-fold less than that of the AR agonists T, R1881, SARM-2F, and T8039 (Figure 7B). 
A

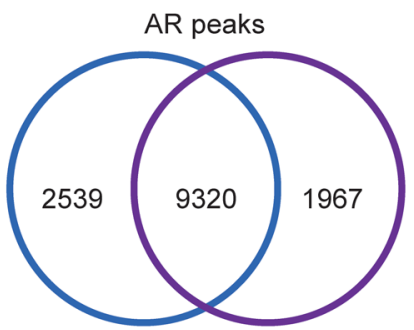

High dose R1881
High dose SARMs

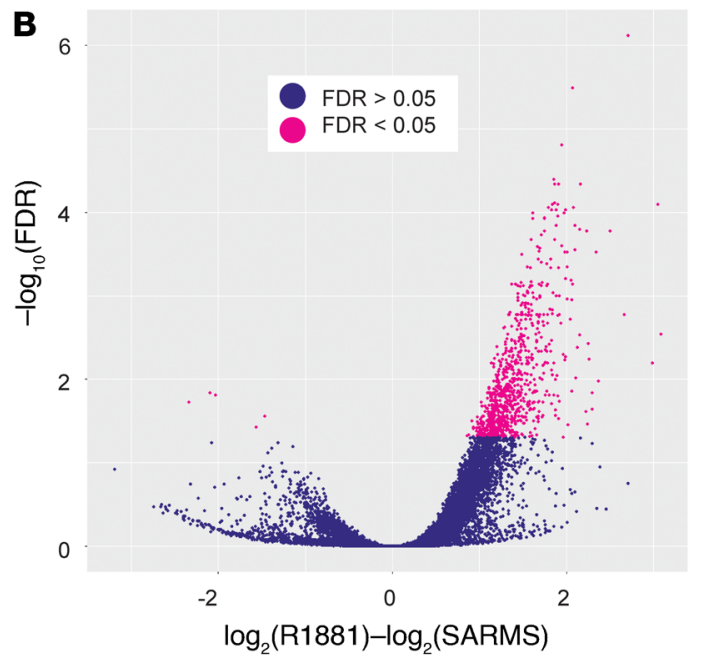

$\log _{2}(\mathrm{R} 1881)-\log _{2}(\mathrm{SARMS})$

C

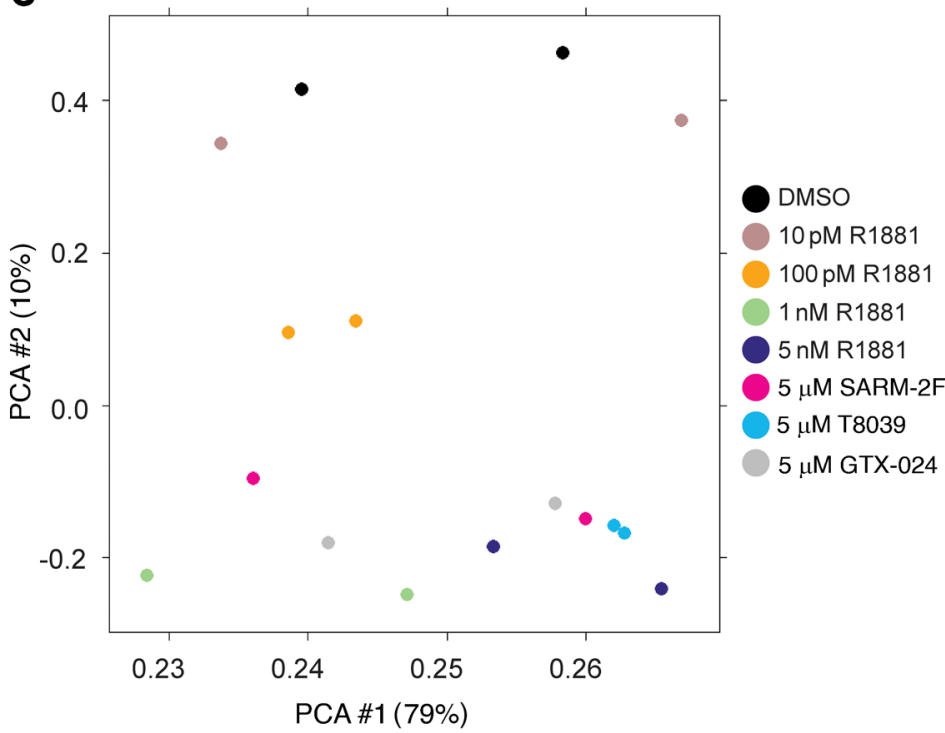

D

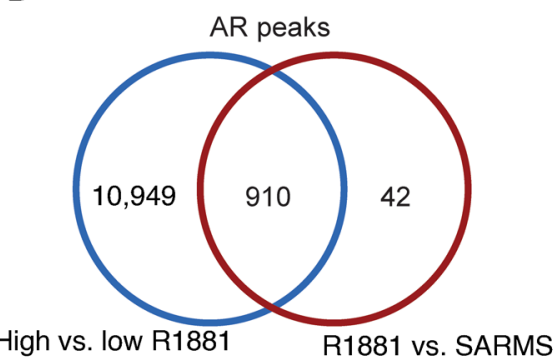

High vs. low R1881

R1881 vs. SARMS

Figure 5. High-dose androgen and high-dose SARM induced AR binding to overlapping sites. (A) Venn diagram of differentially bound sites between high- and low-dose R1881 overlapped with sites differentially bound between high-dose SARMs and low-dose R1881. (B) Volcano plot of differentially bound AR sites between high-dose R1881 and all SARM treatments. (C) PCA plot comparing SARM- and R1881-induced AR cistromes. (D) Overlap of differentially bound AR sites for high- versus low-dose R1881 and high-dose R1881 versus high-dose SARMs.

In contrast to SARM-2F and T8039, GTX-024 and GTX-027 were ineffective at suppressing the growth of 22PC-EP cells, suggesting that SARM responses may also depend on intrinsic cellular factors (Figure 7B). To investigate the lack of growth suppression by GTX-024 and GTX-027 in 22PC-EP cells, we evaluated the ability of these SARMs to influence the expression of the AR-regulated genes FKBP5 and UGT2B17 by qRT-PCR (Supplemental Figure 9, A and B). We found that GTX-024 and GTX-027 were unable to strongly induce FKBP5 expression and only modestly repressed UGT2B17 transcripts in 22PC-EP cells. We then sought to determine whether the GTX compounds differentially induced mutant $\mathrm{AR}^{\mathrm{H} 874 \mathrm{Y}}$ activity. We expressed wild-type AR ( $\left.\mathrm{AR}^{\mathrm{WT}}\right)$ or $\mathrm{AR}^{\mathrm{H} 874 \mathrm{Y}}$ in AR-null PC3 cells and measured FKBP5 expression by qRT-PCR (Supplemental Figure 9, C and D). GTX-024 and GTX-027 induced FKBP5 expression at levels comparable to those of as SARM-2F and T8039 in PC3-AR ${ }^{\mathrm{WT}}$ cells. In contrast, GTX-024 and GTX-027 activated FKBP5 expression in $\mathrm{PC} 3-\mathrm{AR}^{\mathrm{H} 874 \mathrm{Y}}$ cells to a lesser extent than did SARM-2F and T8039. Even so, the GTX compounds still induced the expression of FKBP5 by approximately 20 -fold over vehicle control levels in
PC3-AR ${ }^{\mathrm{H} 87 \mathrm{Y}}$ cells, suggesting that other factors may also play a role in the lack of response of 22PC-EP cells to GTX compounds.

To assess whether the growth-suppressive effects of SARMs were due to their modulation of the AR rather than off-target responses that emerge at high doses, we tested the effects of SARMs on LNCaP-APIPC cells, a LNCaP derivative engineered to lack AR expression (39). The SARMs, steroidal androgens, and ENZ did not influence the growth of this cell line (Figure 7C).

We have previously reported that androgens and the AR upregulate the transcription and expression of the drug transporter SLC35F2 (48). In addition to natural substrates, SLC35F2 mediates the cellular influx of the survivin (aka BIRC5) inhibitor YM155, which results in cell growth arrest and apoptosis (48). Consequently, androgen treatment provides a therapeutic index for treating AR-active cells with YM155 via upregulation of the transporter. We determined that cotreatment of LNCaP cells with SARM-2F and YM155 significantly reduced cell viability, shifting the $\mathrm{IC}_{50}$ of YM155 from $40 \mathrm{nM}$ without SARM-2F to $10 \mathrm{nM}$ with SARM-2F $(P=0.001$; Figure 7D). 
A

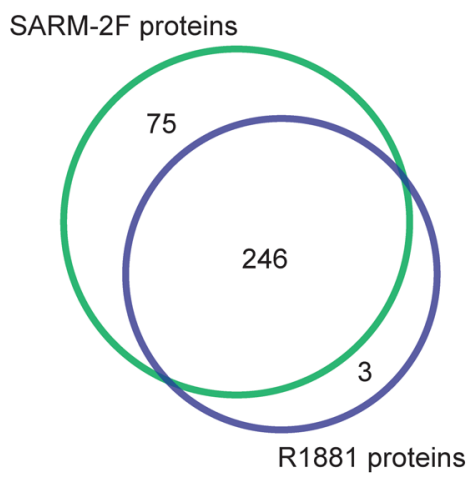

B

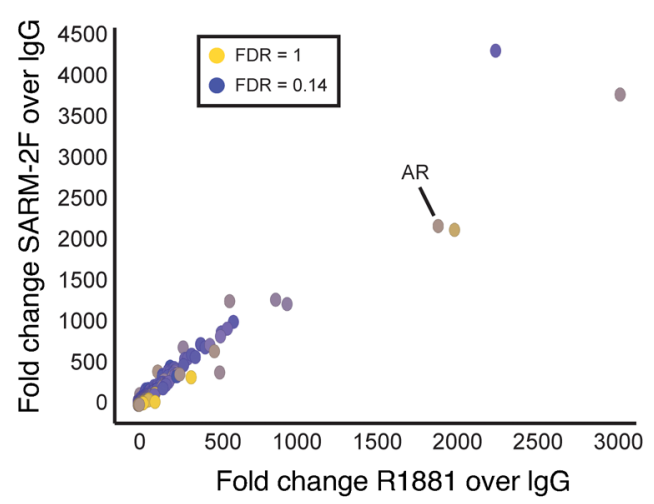

D

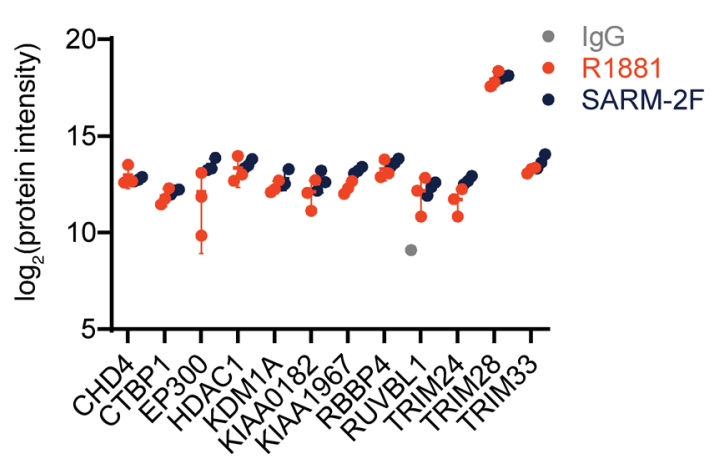

$\mathbf{F}$

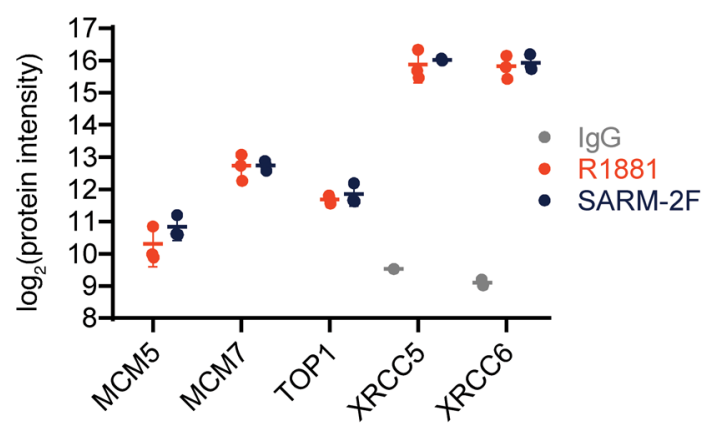

Figure 6. SARMs and steroidal androgens promote analogous AR-cofactor interactions. (A) Venn diagram of the number proteins detected by RIME analysis of AR-bound chromatin complexes for $5 \mu \mathrm{M}$ SARM-2F- and $5 \mu \mathrm{M}$ R1881-treated LNCaPs. FDR $q<0.05(n=3)$. (B) Fold change of AR-bound proteins over IgC for R1881- and SARM-2F-treated samples as detected by RIME $(n=3)$. (C) Protein signal intensity for the AR and AR cofactors involved in luminal prostate differentiation. (D) Transcription factors complexed with the AR detected by RIME. (E) SWI/SNF factors detected in AR complexes. (F) DNA repair and replication factors in AR complexes. $(\mathbf{C}-\mathbf{F}) n=3$. Data represent the mean $\pm \mathrm{SD}$.

SARMs repress PC growth in vivo. To determine whether SARMs can repress PC growth in vivo, we treated 2 PC patient-derived xenograft (PDX) cell lines with SARMs and compared their effects against $\mathrm{T}$. We first performed a dose-finding experiment to confirm that oral administration of SARMs would activate AR signaling. Mice bearing LuCaP 35CR PDX tumors were treated with 15 or $30 \mathrm{mg} / \mathrm{kg}$ T8039 orally, or 100 or $200 \mathrm{mg} / \mathrm{kg}$ SARM-2F orally, or $40 \mathrm{mg} / \mathrm{kg}$ T-cypionate by i.m. injection. After 48 hours, the tumors were resected, RNA was extracted, and KLK3 and FKBP5 transcript levels were quantitated by qRT-PCR. Each SARM sub- stantially increased KLK3 (Supplemental Figure 9E) and FKBP5 (Supplemental Figure 9F) transcripts in the LuCaP 35CR tumors, indicating that SARMs act as agonists in vivo.

We next evaluated the effects of SARMs on growth of the castration-sensitive LuCaP $96 \mathrm{PDX}$ and castration-resistant LuCaP 35CR PDX. After growth to a tumor volume of $150 \mathrm{~mm}^{3}$, mice with LuCaP 35CR tumors were treated 5 days a week with oral doses of $30 \mathrm{mg} / \mathrm{kg}$ T8039 or $100 \mathrm{mg} / \mathrm{kg}$ SARM-2F, or every 2 weeks with 40 $\mathrm{mg} / \mathrm{kg}$ T-cypionate given i.m. Compared with the vehicle-treated controls, SARM-2F and T8039 significantly suppressed the growth 
A
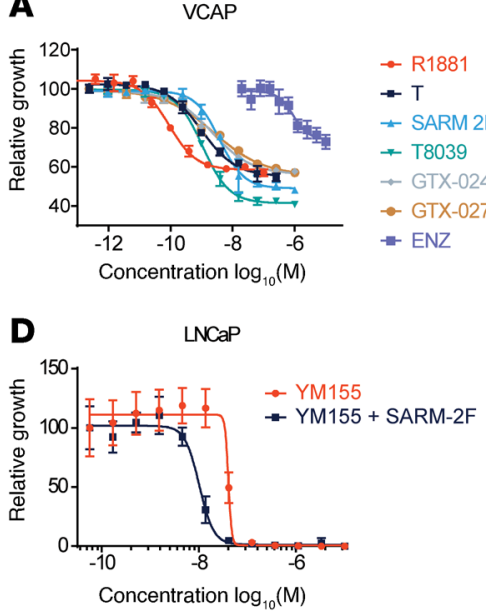

G
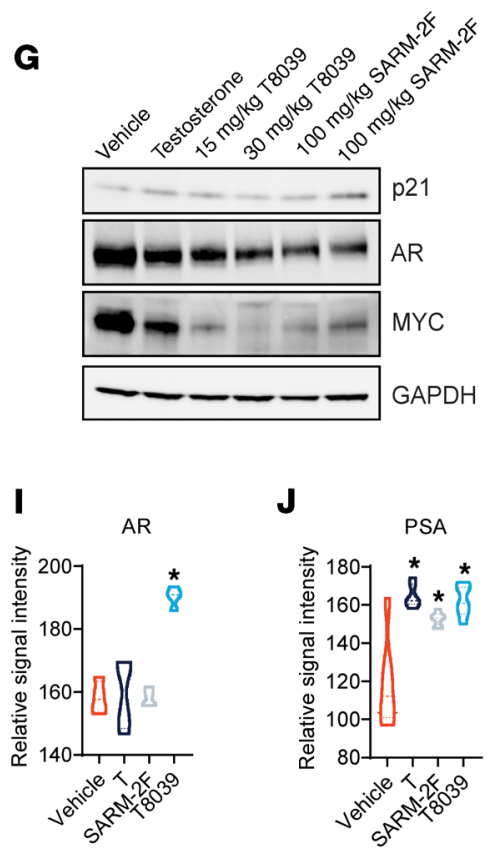

$\mathbf{K}$

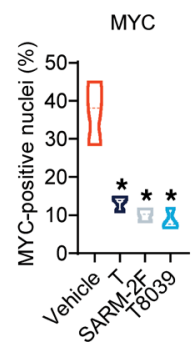

- R1881

$-\mathrm{T}$

- SARM $2 \mathrm{~F}$

- T8039

$\rightarrow$ GTX-024

- GTX-027

- ENZ

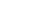
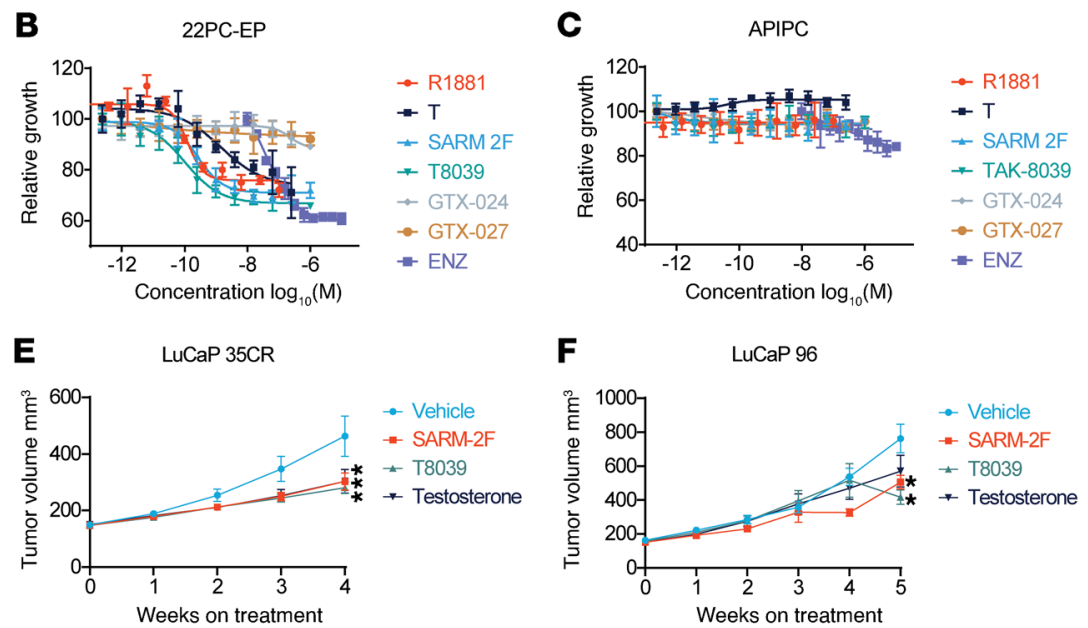

H

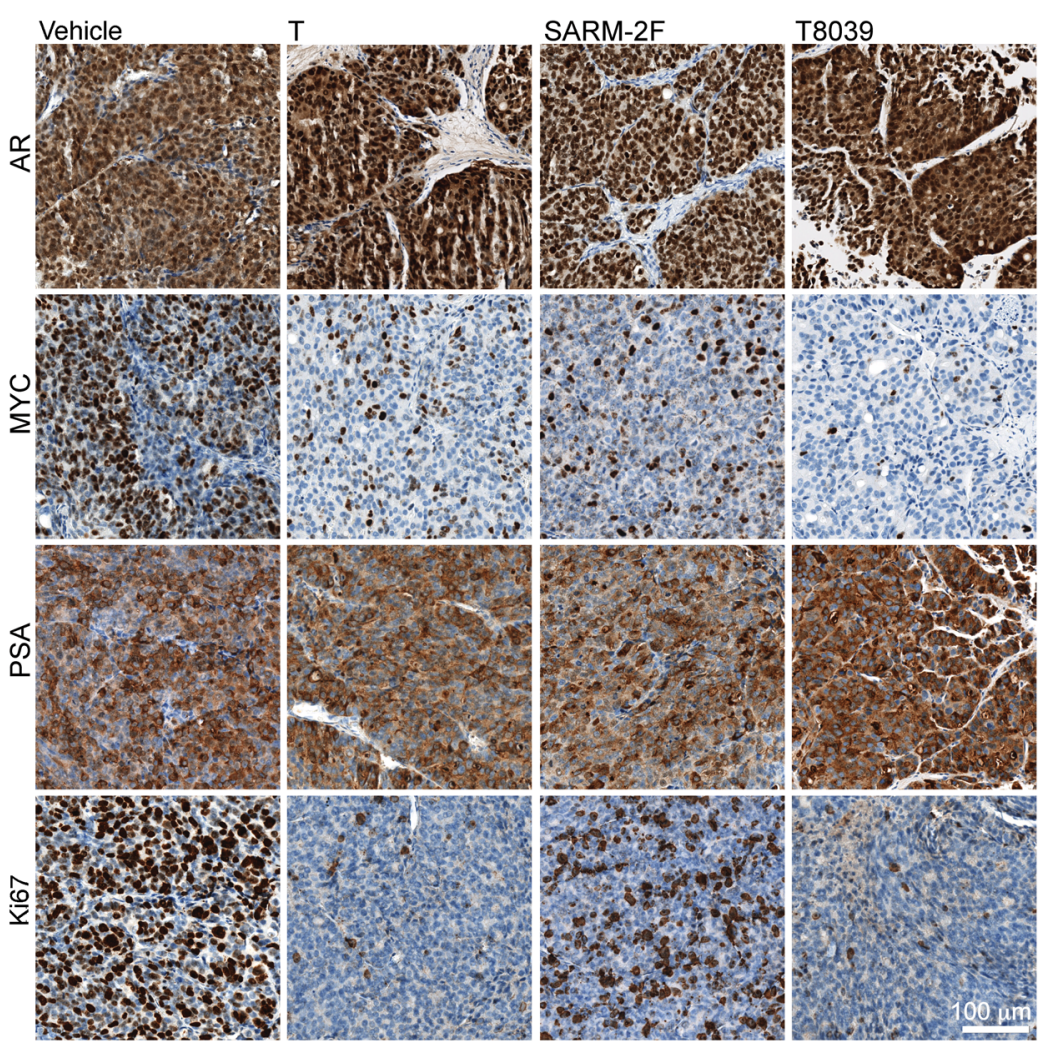

Figure 7. SARMs activate AR signaling and repress PC growth in vitro and in vivo. (A-D) Dose-response curves were generated for the steroidal androgens R1881 and T, the antiandrogen ENZ, the nonsteroidal AR agonists T8039, GTX-024, GTX-027, and SARM-2F for (A) VCaP, (B) 22PC-EP, and (C) APIPC cell lines $(n=4)$. Data represent the mean \pm SD. (D) Dose-response curves for the survivin inhibitor YM155 in LNCaP cells with and without $5 \mu$ M SARM-2F $(n=4)$. Data represent the mean \pm SD. (E) Tumor volume plot of LuCaP 35CR PDXs treated 5 times per week with vehicle $(n=10)$, 100 $\mathrm{mg} / \mathrm{kg} \mathrm{SARM-2F}(n=10)(P=0.045)$, or $30 \mathrm{mg} / \mathrm{kg}$ T8039 $(n=10)(P=0.02)$, or with biweekly $40 \mathrm{mg} / \mathrm{kg}$ i.m. injections of T for $28 \mathrm{days}(n=10)(P=$ 0.047). Data represent the mean \pm SEM. (F) Tumor volume plot of LuCaP 96 PDXs treated 3 times per week with the indicated doses of SARMs: 100 $\mathrm{mg} / \mathrm{kg} \mathrm{SARM-2F}(n=8)(P=0.04)$, or $30 \mathrm{mg} / \mathrm{kg} \mathrm{T} 8039(n=5)(P=0.014)$, or biweekly with i.m. injections of $40 \mathrm{mg} / \mathrm{kg} \mathrm{T}(n=7)(P=0.171)$ or vehicle $(n$ = 7). Data represent the mean \pm SEM. (C) Immunoblots of 35CR PDX lysates for p21, AR, and MYC. Lysates were harvested 48 hours after dosing. (H) IHC images of AR, KLK3, MYC, and Ki67 in 35CR PDX tumors analyzed 48 hours after dosing with vehicle, $40 \mathrm{mg} / \mathrm{kg} \mathrm{T,} 30 \mathrm{mg} / \mathrm{kg} \mathrm{T8039,} \mathrm{or} 100 \mathrm{mg} / \mathrm{kg}$ SARM-2F. Scale bar: $100 \mu \mathrm{m}$. Original magnification, $\times 20$. (I) Relative signal intensity for the AR by IHC $(n=4)$. (J) Relative signal intensity for PSA by IHC $(n=4)$. (K) Percentage of MYC-positive nuclei by IHC $(n=3)$. (L) Ki67-positive staining by IHC $(n=3)$. (I and J) Data represent the mean \pm first and third interquartile range. ${ }^{*} P<0.05$, by 1 -way ANOVA with Dunnett's multiple-comparison test. 
LuCaP 35CR tumors grown in castrated mice: after 4 weeks of treatment, tumor volumes in the control mice averaged $463 \mathrm{~mm}^{3}$, whereas tumors in the SARM-2F-treated mice averaged $303 \mathrm{~mm}^{3}(P$ $=0.045)$. Tumors in the T8039-treated mice averaged $281 \mathrm{~mm}^{3}(P=$ $0.02)$, and tumors in T-treated mice averaged $304 \mathrm{~mm}^{3}(P=0.047$; Figure 7E). LuCaP 96 tumors, grown in intact male mice, were treated 3 times weekly with the same doses of SARMs and T. After 5 weeks, tumors in the vehicle-treated mice averaged $763 \mathrm{~mm}^{3}$, whereas tumors from mice treated with SARM-2F, T8039, or T averaged $506 \mathrm{~mm}^{3}(P=0.04), 418 \mathrm{~mm}^{3}(P=0.014)$, and $571 \mathrm{~mm}^{3}(P=$ 0.171 ) in size, respectively (Figure $7 F)$. Immunoblotting performed on LuCaP 35CR tumors 48 hours after treatment showed reduced MYC and AR expression, but p21 levels were unchanged (Figure 7G). The differences we observed in AR reduction between LNCaP and LuCaP35 may reflect intrinsic differences in AR autoregulation or regulation of AR protein stability. Immunohistochemical analysis confirmed that LuCAP 35CR tumors expressed high levels of the AR protein and that KLK3/PSA expression increased with SARM and T treatment (Figure 7, H-J). SARM and T treatment decreased nuclear MYC expression (Figure 7K) and reduced the number of Ki67-positive cells from $33 \%$ in the vehicle control-treated tumors to $6 \%$ in the T8039-treated tumors $(P<0.001)$ and $17 \%$ in the SARM-F2treated tumors $(P<0.001)$ (Figure $7 \mathrm{~L})$.

\section{Discussion}

AR signaling is critical for the growth and survival of most PCs throughout the entire course of the disease. However, successive treatments with drugs designed to inhibit AR signaling yield diminishing benefits and extensive cross-resistance (49). SAT may counter adaptive cellular mechanisms that contribute to a castration-resistant phenotype. Notably, the dynamic nature of bipolar $\mathrm{T}$ administration further exploits a requirement for adaptive responses to maintain optimal ratios of ligand and receptor that regulate cell survival and proliferation (14). Additionally, mechanisms that enhance AR function in low androgen environments, like $A R$ amplification, may become liabilities when high concentrations of $\mathrm{T}$ activate the growth-suppressive functions of the AR. Unfortunately, the use of $\mathrm{T}$ as a therapeutic is accompanied by pharmacological limitations that preclude the optimal evaluation of AR agonism as a treatment strategy. For example, if cycling high and low AR agonism is an important facet of clinical benefit, it is challenging to rapidly adjust plasma androgen concentrations using the current methods of depot i.m. injections. Given these considerations, discovering a substitute for $\mathrm{T}$ with improved safety, delivery, and control would represent a significant advance in the use of continuous or bipolar AR agonist therapies in the clinic.

In model systems, SARMs have been shown to be potent AR agonists, for example, restoring body and muscle mass of castrated rats (22). The pharmacokinetics of several SARMs have been extensively characterized $(19,22,23,50)$. In terms of AR agonist activity, SARM-2F and GTX-024 have superimposable transcriptional dose responses toward AR-driven reporters: saturating activity was achieved for both SARMs at $10^{-8} \mathrm{M}$ (19). Similar dose-dependent transcriptional responses were observed in primary human skeletal muscle and prostate epithelial cells (22). While SARM abuse has been reported, and toxicities have been associated with adulterated or misrepresented products obtained via internet vendors (51), clinical studies using pure clinical-grade SARM compounds have demonstrated excellent safety profiles. For example, a phase II clinical study of GTX-024 (enobosarm) for cancer-induced muscle wasting did not report significant adverse events (33).

Although the drug-like properties of SARMs are well supported by the literature, it was unknown whether SARMs, being partial agonists, would activate the AR potently enough to engage a growth-suppressive and differentiating transcriptional program in PC. In this study, we tested the hypothesis that nonsteroidal SARMs match the efficacy of steroidal AR agonists in suppressing the growth of castration-resistant PC. To this end, we evaluated SARMs in vitro and in vivo to establish their growth-suppressive activities in PC models. Like steroidal androgens, we found that SARMs induced the expression of luminal differentiation genes, blocked entry into the S phase, and repressed MYC and FOXM1 levels. Finally, by comparing transcriptomes, AR cistromes, and AR cofactor profiles, we demonstrated that SARMs recapitulated the global molecular effects of steroidal AR agonists.

The most unexpected result from this study was the lack of meaningful differences between certain SARMs and steroidal agonists in PC cells. Previous studies have suggested that SARMs and steroidal androgens differentially recruit cofactors $(21,43)$. However, there does not appear to be a consistent set of cofactors responsible for the "androgenic" versus the "anabolic" effects. Part of this confusion may be due to the vast diversity of SARMs in both molecular scaffolds and the potency of their agonism. In our study, we found that the SARMs LGD-4033 and RAD140 were unable to suppress the growth of PC cells. Interestingly, GTX-024 and GTX-027 activated the AR sufficiently to suppress the growth of LNCaP and VCAP cells, but not 22PC-EP cells. These data suggest that the effect of a SARM depends on the cell-intrinsic context of AR signaling, like $A R$ mutation or amplification status, as well as on the properties of the SARM itself.

Currently, it is difficult to predict whether a particular SARM can activate the AR sufficiently to suppress the PC growth $(45,46)$. Given that certain SARMs behave as partial agonists in vitro and in benign tissues, and yet fully recapitulate AR activity in PC models, the switch from partial agonist to full agonist may reflect genomic and epigenomic alterations in prostate carcinomas, where cistrome reprogramming influences the development of castration resistance. These mechanisms should be further studied in the context of SARM sensitivity to predict likely responders.

The effects of bipolar AR activation were not evaluated in this study. Preventing adaptation to a static, low or high androgen environment may be crucial, for long-term suppression of tumor growth. Understanding changes in gene expression, physiology, and the epigenetics of cells exposed to a changing androgen environment may help improve therapy by identifying feedback mechanisms that can be further exploited. Additionally, the current 28-day treatment cycle of BAT may not be the most effective. Given the control afforded by the drug-like properties of SARMs, shorter or longer treatment intervals could be evaluated to optimize the bipolar AR for the greatest effect.

Future studies into the use of nonsteroidal agonists to suppress the growth of PC should explore strategies that augment the activation of the AR. One avenue could be to evaluate nonsteroidal, full AR agonists. Safety concerns notwithstanding, full agonists may 
be more effective replacements for steroidal agonists by potently inducing AR signaling, regardless of the cellular context, and so could induce the same systemic effects as those seen with T. Other possibilities include cotherapeutic approaches to directly activate the N-terminal domain of the AR or stabilize AR levels (52-54).

Our findings strongly support further exploration of SARMs for the treatment of CRPC. The nonsteroidal compounds SARM2F, T8039, GTX-024, and Cpd26 potently induced AR activity, repressed MYC signaling, and suppressed the growth of PC cells. SARMs compared favorably to steroidal androgens by regulating a largely overlapping set of genes and recruiting the same set of AR cofactors to chromatin. Since SARMs have already been tested in a variety of clinical settings for other health conditions, the path to implementing them in the clinic as a CRPC therapy could be accelerated. Given their convenient mode of delivery, favorable safety profile, and potential to improve general health, SARMs present an attractive therapeutic option.

\section{Methods}

In vitro cell cultures. $\mathrm{LNCaP}$ cells were obtained from American Type Culture Collection (ATCC) (CRL-1740) and cultured in RPMI-1640 (phenol red-free) (Gibco, Thermo Fisher Scientific, catalog 11835030) supplement with 10\% FBS (Gibco, Thermo Fisher Scientific, catalog 10437-02). VCAP cells were obtained from ATCC (CRL-2876) and grown in DMEM/F12 (phenol red-free) (Gibco, Thermo Fisher Scientific, catalog 21041025) with 10\% FBS. PC-3 cells were also obtained from ATCC (CRL-1435) and grown in DMEM/F12 (phenol red-free) (Gibco, Thermo Fisher Scientific, catalog 21041025) with 10\% FBS. 22PC-EP, a gift from the laboratory of Charles Sawyers at Memorial Sloan Kettering Cancer Center (New York, New York, USA), were grown in RPMI-1640 and 10\% FBS. Cultures were maintained in a 5\% $\mathrm{CO}_{2}$ incubator at $37^{\circ} \mathrm{C}$. Cells were tested for mycoplasma and validated by the DNA Diagnostic Center (DDC) (Fairfield, Ohio, USA) and were used within 20 passages of receipt.

Dose-response assays. Cells were plated at 5000 cells per well in black-walled tissue culture-coated plates (Corning, catalog 3764BC) in normal growth media. Cells were incubated with a dose range of drugs dissolved in media $(n=4)$ to a final volume of $100 \mu \mathrm{L}$ per well. After 4 days, $30 \mu \mathrm{L} /$ well Celltiter-Glo (Promega, catalog G7572) was added, and luminescence was measured on a Synergy $\mathrm{H} 1$ microplate reader (BioTek). The following drugs were used in the study: enzalutamide (Selleckchem, catalog S1250); GTX-024 (Selleckchem, catalog MK-2866); GTX-027 (a gift from James Dalton, University of Michigan, Ann Arbor, Michigan, USA); SARM-2F and T8039 (2-chloro-4-[(2S,3S)-3-hydroxy-2-(trifluoroethyl)-5-oxopyrrolidin-1-yl]benzonitrile; gifts from Takeda, Kanagawa, Japan); Cpd26 (a gift from Novartis, Basel, Switzerland); RAD-140 (Selleckchem, catalog S5275); LGD-4033 (Selleckchem, catalog S8822); YM155 (Selleckchem, catalog S1130); and R1881 (PerkinElmer, catalog NLP005005mg).

$q R T$-PCR. Cells $\left(1 \times 10^{6}\right)$ were plated in 6-well plates in normal growth media, and after 24 hours, cells were dosed at the indicated levels and cultured for 48 hours. Cells were then harvested using an RNeasy kit (QIAGEN, catalog 74104). cDNA was made using SuperScript II (Thermo Fisher Scientific, catalog 18064014). Expression levels were measured using Power Sybr Green (Applied Biosystems, catalog 4367659) and on a Bio-Rad CFX384 real-time system. The primers used are listed in Supplemental Table 2.
RNA-Seq. RNA was extracted as described above. RNA ( $1 \mu \mathrm{g})$ was processed into a library using the TruSeq Stranded mRNA LT Sample Prep Kit (Illumina). Libraries were sequenced using the HiSeq 2500 (Illumina) with 50 bp paired-end reads. Reads were mapped and aligned to the human genome hg38 using TopHat, version 2 (55). Differentially expressed genes were determined using GenomicAlignments (56) and edgeR (57). A gene expression cutoff of 1 count per million in at least 2 samples was used. Pathway analysis was performed using GSEA (58) and GSVA (59).

Cell-cycle analysis. Cells $\left(1 \times 10^{6}\right)$ were plated in 6-well plates and cultured for 48 hours with the indicated drugs. Cell-cycle analysis was performed using a Click-iT EdU Alexa Fluor 488 Flow Cytometry Assay Kit (Thermo Fisher Scientific, catalog C10420) as an S-phase marker and FxCycle Violet Stain (Thermo Fisher Scientific, catalog F10347) as a DNA content stain.

Immunoblotting and IHC. Lysates were run on NuPAGE 4\%-12\% Bis-Tris gels (Thermo Fisher Scientific, catalog NP0321) in MOPS buffer (Thermo Fisher Scientific, catalog NP0001). Proteins were bound to nitrocellulose membranes (Thermo Fisher Scientific, catalog LC2000) in NuPAGE transfer buffer (Thermo Fisher Scientific, catalog NP0006) according to the manufacturer's instructions. The following antibodies from Cell Signaling Technology were used: GAPDH (catalog 2118L); $\gamma$-H2AX (catalog 9718T); c-Myc (catalog 13987s); p16 (catalog 92803s); p21 (catalog 2947s); and FOXM1 (catalog 5436s). Uncut immunoblots can be viewed in the online supplemental material. IHC was performed as previously described (39). Automated IHC was performed using a DISCOVERY ULTRA instrument (Ventana Medical Systems). Tissue sections were cut at a thickness of $4 \mu \mathrm{m}$ and mounted onto positively charged slides. Onboard deparaffinization was performed using DISCOVERY Wash Buffer (Ventana, catalog 950-510) followed by heat-induced epitope retrieval with DISCOVERY CC1 solution (Ventana, catalog 950-224). The sections were incubated with the following primary monoclonal antibodies: AR (Cell Signaling Technology, D6F11) at 1:100; c-MYC (Abcam, Y69) at 1:50; PSA (Leica, 35h9) at 1:50; or Ki67 (Thermo Fisher Scientific, SP6) at 1:100. The PSA and Ki67 slides were incubated with anti-mouse IgGs (Abcam, M111-2) at 1:200 prior to application of the secondary antibodies. All slides were incubated with the secondary antibody on a DISCOVERY anti-rabbit HQ-HRP System (Ventana, catalog 760-4820). The c-Myc signal was further amplified using the DISCOVERY AMP kit (Ventana, catalog 760-052). The antibodies were detected with a ChromoMap DAB Detection Kit (Ventana, catalog 760-159). Sections were counterstained using Hematoxylin II (Ventana, catalog 790-2208), followed by Bluing Reagent (Ventana, catalog 760-2037). The slides were scanned using the Ventana DP 200 instrument (Ventana Medical Systems).

$C U T \& R U N$. CUT\&RUN was performed on $5 \times 10^{5} \mathrm{LNCaP}$ cells cultured for 4 hours in the specified treatment after being cultured for 48 hours in RPMI-1640 with $10 \%$ charcoal-stripped serum (Thermo Fisher Scientific, catalog SH3006803). Each condition was performed in duplicate. Cells were processed as previously described (60) using pAG-mnase and the low-salt protocol with the AR antibody (MilliporeSigma, catalog 06-680). Sequencing libraries were prepared using a MicroPlex Library Preparation Kit, version 2 (Diagenode, catalog C05010014) and sequenced using the Illumina HiSeq 2500 in rapid run mode with 50 bp paired-end reads. Reads were aligned to 
the hg19 genome using Bowtie 2 (61). Peaks were called using MACS2 (62). Gene annotations were performed using HOMER (63) and the ChIPSeeker R package (64). Differentially bound peaks were determined using the DiffBind R package (65). Peaks were visualized using IGV (66). DeepTools 3.3.0 was used to calculate matrices and plot heatmaps for binding sites (67).

MARCoNI assay. The MARCoNI assay was performed by PamGene International. Saturating doses $(10 \mu \mathrm{M})$ of ligands were compared with DHT and DMSO using the 154 coregulator PamChip array (PamGene, catalog 88101) and the AR LBD (LBD-GST, Invitrogen, Thermo Fisher Scientific, catalog A15675). Each compound was tested with 3 replicates in agonist mode versus solvent ( $2 \% \mathrm{DMSO})$.

RIME. LNCaP cells were cultured for 48 hours in $10 \%$ charcoal-stripped serum media and then incubated for 4 hours with $5 \mu \mathrm{M}$ SARM-2F or $5 \mathrm{nM}$ R1881. Cells were fixed, permeabilized, and immunoprecipitated as previously published (47). Extracts were sonicated using a Covaris M220 sonicator. Chromatin was immunoprecipitated using a 1:1 mixture of the antibodies AR (MilliporeSigma, catalog 06-680) and AR (Active Motif, catalog 39781). Data were analyzed using Proteome Discover (Thermo Fisher Scientific). Results were further filtered using the CRAPome repository to remove the top 250 proteins bound to GFP in 293 cells (68). Ribosomal and histone proteins were also removed from the analysis. Gene lists identified by mass spectrometry were annotated using Panther GO (69).

Patient-derived xenograft experiments. NOD/SCID $\gamma$ (NSG) mice (The Jackson Laboratory, 005557), aged 6-8 weeks, were implanted s.c. with either LuCaP 35CR or LuCaP 96 PC PDXs to yield 8-10 evaluable tumors per treatment arm. LuCaP 35CR and LuCaP 96 are, respectively, castration-resistant and castration-sensitive PC PDX models expressing the WT AR that were established by the University of Washington Medical Center (UWMC) Genitourinary Biorepository as previously described (70). Mice that received LuCaP 35CR were castrated 2 weeks prior to implantation with tumor pieces. Tumors were measured 3 times per week, and tumor volume was calculated as: length $\times\left(\right.$ width $\left.^{2}\right) / 2$. When tumors reached $150 \mathrm{~mm}^{3}$, mice were randomized to vehicle ( $0.5 \%$ methylcellulose) or treatment $(30 \mathrm{mg} / \mathrm{kg}$ T8039 5×/week p.o., 100 mg/kg SARM-2F 5×/week p.o., or 40 mg/ $\mathrm{kg}$ T-cypionate via biweekly intramuscular injection) arms. The mice were monitored until tumors reached approximately $1000 \mathrm{~mm}^{3}$ in size (end of study [EOS]), at which point the mice were euthanized according to institutional protocols, and tumors were harvested for analysis.

Statistics. For the RNA-Seq experiments, FDR $q$ values of differential genes and gene set expression were generated using algorithms for edgeR and GSEA, respectively. RIME data were processed using algorithms internal to Proteome Discover. MARCoNI assay data points were compared using the Student's $t$ test. CUT\&RUN motif $P$ and $q$ values (Benjamini) were generated using HOMER and differential binding FDR $q$ values using algorithms internal to DiffBind. qRT-PCR data were compared using 1-way ANOVA with Dunnett's multiple-comparison test on fold-change values calculated using the $\Delta \Delta \mathrm{Ct}$ method. For growth assays, evaluation of $\mathrm{IC}_{50}$ and drug synergies was done using the extra sum-of-squares $F$ test in GraphPad Prism 8 (GraphPad Software). Individual dose points were evaluated using the Student's $t$ test. Dose points for in vivo growth assays were evaluated using 1-way ANOVA with Dunnett's multiple-comparison test. Significance for IHC analysis was calculated using 1-way ANOVA with Dunnett's multiple-comparison test. Significance for cell-cycle analyses was calculated using 1-way ANOVA with Sidak's multiple-comparison test in GraphPad Prism 8.0.1.

Study approval. All animal experiments were performed in accordance with protocols approved by the IACUC of the Fred Hutchinson Cancer Research Center and following the recommendations in the NIH's Guide for the Care and Use of Laboratory Animals (National Academies Press, 2011). This study did not involve human subjects.

\section{Author contributions}

MDN and PSN designed the study and wrote the manuscript. SRP provided study design feedback and AR expertise. MPM, DHJ, and NDS provided technical guidance for the CUT\&RUN assays. $\mathrm{RD}$ and $\mathrm{TH}$ performed IHC staining and imaging. $\mathrm{MCH}$ provided IHC guidance, performed IHC analysis, and provided guidance on RIME design. A Corella, MDN, and IMC contributed to RNA-Seq analysis. A Corella, LB, and MDN performed RIME. CP and MDN performed growth assays. HM, AB, and MDN performed qRTPCR experiments. SBF provided guidance on cell-cycle analysis. JTD provided GTX-027 and SARM expertise. EAM provided PDX experiment design guidance, study design guidance, and manuscript feedback. EC generated the PDX cell lines. LA and A Christiani conducted PDX experiments.

\section{Acknowledgments}

We thank Steve Balk, Henry Long, Myles Brown, and members of the Nelson laboratory for helpful advice and criticism. We thank Hansjoerg Keller from Novartis for providing Cpd26 and Atsushi Hasuoka, Katsuji Aikawa, Megumi Morimoto, and Takahito Hara from Takeda Pharmaceutical Company Ltd. for providing SARM-2F and T8039. We thank Steven Henikoff for providing the pAG-MNase enzyme for the CUT\&RUN studies. We gratefully acknowledge research support from the NIH (R21CA230138, P30CA015704-40, P50CA097186, P01CA163227, and R01 CA234715); a Congressionally Directed Medical Research Program (CDMRP) award (PC170431); the Prostate Cancer Foundation; and the Institute for Prostate Cancer Research. The graphical abstract was created in BioRender.

Address correspondence to: Peter S. Nelson, Division of Human Biology, Fred Hutchinson Cancer Research Center, Mailstop E2-152, 1100 Fairview Ave. N., Seattle, Washington 98109-1024, USA. Phone: 206.616.0103; Email: pnelson@fredhutch.org.

\footnotetext{
1. Nelson PS, et al. The program of androgen-responsive genes in neoplastic prostate epithelium. Proc Natl Acad Sci U S A. 2002;99(18):11890-11895.

2. Heinlein CA, Chang C. Androgen receptor in prostate cancer. Endocr Rev. 2004;25(2):276-308.

3. Cunha GR, et al. The endocrinology and developmental biology of the prostate. Endocr Rev. 1987;8(3):338-362.
}

4. Nelson PS. Molecular states underlying androgen receptor activation: a framework for therapeutics targeting androgen signaling in prostate cancer. JClin Oncol. 2012;30(6):644-646.

5. Watson PA, et al. Emerging mechanisms of resistance to androgen receptor inhibitors in prostate cancer. Nat Rev Cancer. 2015;15(12):701-711.

6. de Bono JS, et al. Abiraterone and increased sur- vival in metastatic prostate cancer. N Engl J Med. 2011;364(21):1995-2005.

7. Scher HI, et al. Increased survival with enzalutamide in prostate cancer after chemotherapy. $N$ Engl JMed. 2012;367(13):1187-1197.

8. Higano CS. Side effects of androgen deprivation therapy: monitoring and minimizing toxicity. Urology.2003;61(2 Suppl 1):32-38. 
9. Davis ID, et al. Enzalutamide with standard firstline therapy in metastatic prostate cancer. $N$ Engl JMed. 2019;381(2):121-131.

10. Kokontis JM, et al. Progression of LNCaP prostate tumor cells during androgen deprivation: hormone-independent growth, repression of proliferation by androgen, and role for $\mathrm{p} 27 \mathrm{Kip} 1$ in androgen-induced cell cycle arrest. Mol Endocrinol. 1998;12(7):941-953.

11. Umekita Y, et al. Human prostate tumor growth in athymic mice: inhibition by androgens and stimulation by finasteride. Proc Natl Acad Sci US A. 1996;93(21):11802-11807.

12. Teply BA, et al. Bipolar androgen therapy in men with metastatic castration-resistant prostate cancer after progression on enzalutamide: an open-label, phase 2, multicohort study. Lancet Oncol. 2018;19(1):76-86.

13. Markowski MC, et al. Extreme responses to immune checkpoint blockade following bipolar androgen therapy and enzalutamide in patients with metastatic castration resistant prostate cancer. Prostate. 2020;80(5):407-411.

14. Schweizer MT, et al. Effect of bipolar androgen therapy for asymptomatic men with castration-resistant prostate cancer: results from a pilot clinical study. Sci Transl Med. 2015;7(269):269ra2.

15. Grech A, et al. Adverse effects of testosterone replacement therapy: an update on the evidence and controversy. Ther Adv Drug Saf. 2014;5(5):190-200.

16. Achar S, et al. Cardiac and metabolic effects ofanabolic-androgenic steroid abuse on lipids, blood pressure, left ventricular dimensions, and rhythm. Am J Cardiol. 2010;106(6):893-901.

17. Vigen $\mathrm{R}$, et al. Association of testosterone therapy with mortality, myocardial infarction, and stroke in men with low testosterone levels. JAMA. 2013;310(17):1829-1836.

18. Basaria S, et al. Adverse events associated with testosterone administration. N Engl J Med. 2010;363(2):109-122.

19. Morimoto M, et al. A selective androgen receptor modulator SARM-2f activates androgen receptor, increases lean body mass, and suppresses blood lipid levels in cynomolgus monkeys. Pharmacol Res Perspect. 2020;8(1):e00563.

20. Christiansen AR, et al. Selective androgen receptor modulators: the future of androgen therapy? Transl Androl Urol. 2020;9(Suppl 2):S135-S148.

21. Narayanan R, et al. Development of selective androgen receptor modulators (SARMs). Mol Cell Endocrinol. 2018;465:134-142.

22. Morimoto M, et al. Amelioration of sexual behavior and motor activity deficits in a castrated rodent model with a selective androgen receptor modulator SARM-2f. PLoS One. 2017;12(12):e0189480.

23. Morimoto $\mathrm{M}$, et al. Prevention of body weight loss and sarcopenia by a novel selective androgen receptor modulator in cancer cachexia models. Oncol Lett. 2017;14(6):8066-8071.

24. Piatnitski Chekler EL, et al. 1-(2-Hydroxy-2-methyl-3-phenoxypropanoyl) indoline-4-carbonitrile derivatives as potent and tissue selective androgen receptor modulators. JMed Chem. 2014;57(6):2462-2471.
25. Basaria S, et al. The safety, pharmacokinetics, and effects of LGD-4033, a novel nonsteroidal oral, selective androgen receptor modulator, in healthy young men. J Gerontol A Biol Sci Med Sci. 2013;68(1):87-95.

26. Gao W, et al. Pharmacokinetics and pharmacodynamics of nonsteroidal androgen receptor ligands. Pharm Res. 2006;23(8):1641-1658.

27. Ullrich T, et al. 3-alkoxy-pyrrolo[1,2-b]pyrazolines as selective androgen receptor modulators with ideal physicochemical properties for transdermal administration. JMed Chem. 2014;57(17):7396-7411.

28. Clark RV, et al. Safety, pharmacokinetics and pharmacological effects of the selective androgen receptor modulator, GSK2881078, in healthy men and postmenopausal women. Br J Clin Phar macol. 2017;83(10):2179-2194.

29. Nyquist MD, et al. Molecular determinants of response to high-dose androgen therapy in prostate cancer. JCI Insight. 2019;4(19):129715.

30. Hedayati M, et al. Androgen deprivation followed by acute androgen stimulation selectively sensitizes AR-positive prostate cancer cells to ionizing radiation. Clin Cancer Res. 2016;22(13):3310-3319.

31. Lam HM, et al. Durable response of enzalutamide-resistant prostate cancer to supraphysiological testosterone is associated with a multifaceted growth suppression and impaired DNA damage response transcriptomic program in patient-derived xenografts. Eur Urol. 2020;77(2):144-155.

32. Chatterjee $P$, et al. Supraphysiological androgens suppress prostate cancer growth through androgen receptor-mediated DNA damage. JClin Invest. 2019;129(10):4245-4260.

33. Dobs AS, et al. Effects of enobosarm on muscle wasting and physical function in patients with cancer: a double-blind, randomised controlled phase 2 trial. Lancet Oncol. 2013;14(4):335-345

34. Narayanan R, et al. Selective androgen receptor modulators (SARMs) negatively regulate triple-negative breast cancer growth and epithelial:mesenchymal stem cell signaling. PLoS One 2014;9(7):e103202.

35. Yuan Y, et al. A phase II clinical trial of pembrolizumab and enobosarm in patients with androgen receptor-positive metastatic triple-negative breast cancer. Oncologist. 2021;26(2):99-e217.

36. Nyquist MD, et al. Combined TP53 and RB1 loss promotes prostate cancer resistance to a spectrum of therapeutics and confers vulnerability to replication stress. Cell Rep. 2020;31(8):107669.

37. Kregel S, et al. Acquired resistance to the second-generation androgen receptor antagonist enzalutamide in castration-resistant prostate cancer. Oncotarget. 2016;7(18):26259-26274.

38. Dalton JT, et al. The selective androgen receptor modulator GTx-024 (enobosarm) improves lean body mass and physical function in healthy elderly men and postmenopausal women: results of a double-blind, placebo-controlled phase II trial. J Cachexia Sarcopenia Muscle. 2011;2(3):153-161.

39. Bluemn EG, et al. Androgen receptor pathway-independent prostate cancer is sustained through FGF signaling. Cancer Cell. 2017;32(4):474-489.

40. Chuu CP, et al. Androgen suppresses proliferation of castration-resistant LNCaP 104-R2 prostate cancer cells through androgen receptor, Skp2, and c-Myc. Cancer Sci. 2011;102(11):2022-2028.

41. Sadasivam S, DeCaprio JA. The DREAM complex: master coordinator of cell cycle-dependent gene expression. Nat Rev Cancer. 2013;13(8):585-595.

42. Krishnan V, et al. Development of a selective androgen receptor modulator for transdermal use in hypogonadal patients. Andrology. 2018;6(3):455-464

43. Baek SH, et al. Ligand-specific allosteric regulation of coactivator functions of androgen receptor in prostate cancer cells. Proc Natl Acad Sci U S A. 2006;103(9):3100-3105.

44. Prekovic S, et al. The effect of F877L and T878A mutations on androgen receptor response to enzalutamide. Mol Cancer Ther. 2016;15(7):1702-1712.

45. Sathya G, et al. Pharmacological uncoupling of androgen receptor-mediated prostate cancer cell proliferation and prostate-specific antigen secretion. Cancer Res. 2003;63(22):8029-8036.

46. Kazmin D, et al. Linking ligand-induced alterations in androgen receptor structure to differential gene expression: a first step in the rational design of selective androgen receptor modulators. Mol Endocrinol. 2006;20(6):1201-1217.

47. Mohammed H, et al. Rapid immunoprecipitation mass spectrometry of endogenous proteins (RIME) for analysis of chromatin complexes. Nat Protoc. 2016;11(2):316-326

48. Nyquist MD, et al. Exploiting AR-regulated drug transport to induce sensitivity to the survivin inhibitor YM155. Mol Cancer Res. 2017;15(5):521-531.

49. de Bono JS, et al. Antitumour activity and safety of enzalutamide in patients with metastatic castration-resistant prostate cancer previously treated with abiraterone acetate plus prednisone for $\geq$ 24 weeks in Europe. Eur Urol. 2018;74(1):37-45.

50. Coss CC, et al. Pharmacokinetic drug interactions of the selective androgen receptor modulator GTx-024(Enobosarm) with itraconazole, rifampin, probenecid, celecoxib and rosuvastatin. Invest New Drugs. 2016;34(4):458-467.

51. Van Wagoner RM, et al. Chemical composition and labeling of substances marketed as selective androgen receptor modulators and sold via the internet. JAMA. 2017;318(20):2004-2010.

52. Ueda T, et al. Activation of the androgen receptor $\mathrm{N}$-terminal domain by interleukin-6 via MAPK and STAT3 signal transduction pathways. J Biol Chem. 2002;277(9):7076-7085.

53. Chen S, et al. Androgen receptor phosphorylation and stabilization in prostate cancer by cyclin-dependent kinase 1. Proc Natl Acad Sci U S A. 2006;103(43):15969-15974.

54. Kohler A, et al. A hormone-dependent feedback-loop controls androgen receptor levels by limiting MID1, a novel translation enhancer and promoter of oncogenic signaling. Mol Cancer. 2014;13:146

55. Kim D, et al. TopHat2: accurate alignment of transcriptomes in the presence of insertions, deletions and gene fusions. Genome Biol. 2013;14(4):R36.

56. Lawrence $M$, et al. Software for computing and annotating genomic ranges. PLoS Comput Biol. 2013;9(8):e1003118.

57. Robinson MD, et al. edgeR: a Bioconductor 
package for differential expression analysis of digital gene expression data. Bioinformatics. 2010;26(1):139-140.

58. Subramanian A, et al. Gene set enrichment analysis: a knowledge-based approach for interpreting genome-wide expression profiles. Proc Natl Acad Sci U S A. 2005;102(43):15545-15550.

59. Hanzelmann $S$, et al. GSVA: gene set variation analysis for microarray and RNA-seq data. $B M C$ Bioinformatics. 2013;14:7.

60. Meers MP, et al. Improved CUT\&amp;RUN chromatin profiling tools. Elife. 2019;8:e46314.

61. Langmead B, Salzberg SL. Fast gappedread alignment with Bowtie 2. Nat Methods. 2012;9(4):357-359.
62. Zhang Y, et al. Model-based analysis of ChIP-Seq (MACS). Genome Biol. 2008;9(9):R137.

63. Heinz S, et al. Simple combinations of lineage-determining transcription factors prime cis-regulatory elements required for macrophage and B cell identities. Mol Cell. 2010;38(4):576-589.

64. Yu G, et al. ChIPseeker: an R/Bioconductor package for ChIP peak annotation, comparison and visualization. Bioinformatics. 2015;31(14):2382-2383.

65. Ross-Innes CS, et al. Differential oestrogen receptor binding is associated with clinical outcome in breast cancer. Nature. 2012;481(7381):389-393.

66. Robinson JT, et al. Integrative genomics viewer. Nat Biotechnol. 2011;29(1):24-26.
67. Ramirez F, et al. deepTools2: a next generation web server for deep-sequencing data analysis. Nucleic Acids Res. 2016;44(W1):W160-W165.

68. Mellacheruvu D, et al. The CRAPome: a contaminant repository for affinity purification-mass spectrometry data. Nat Methods. 2013;10(8):730-736.

69. Mi H, et al. PANTHER in 2013: modeling the evolution of gene function, and other gene attributes, in the context of phylogenetic trees. Nucleic Acids Res. 2013;41(Database issue):D377-D386.

70. Nguyen HM, et al. LuCaP prostate cancer patient-derived xenografts reflect the molecular heterogeneity of advanced disease and serve as models for evaluating cancer therapeutics. Prostate. 2017;77(6):654-671. 